\title{
Sum rules and dualities for generalized parton distributions: is there a holographic principle?
}

\author{
K. Kumerički ${ }^{a}$, D. Müller ${ }^{b}$, K. Passek-Kumerički ${ }^{c}$ \\ ${ }^{a}$ Department of Physics, Faculty of Science, University of Zagreb \\ P.O.B. 331, HR-10002 Zagreb, Croatia \\ ${ }^{b}$ Institut für Theoretische Physik II, Ruhr-Universität Bochum \\ D-44780 Bochum, Germany \\ ${ }^{c}$ Theoretical Physics Division, Rudjer Bošković Institute \\ P.O.Box 180, HR-10002 Zagreb, Croatia
}

\begin{abstract}
To leading order approximation, the physical content of generalized parton distributions (GPDs) that is accessible in deep virtual electroproduction of photons or mesons is contained in their value on the cross-over trajectory. This trajectory separates the $t$-channel and $s$-channel dominated GPD regions. The underlying Lorentz covariance implies a correspondence between these two regions through their relation to GPDs on the cross-over trajectory. This point of view leads to a family of GPD sum rules which are a quark analogue of finite energy sum rules and it guides us to a new phenomenological GPD concept. As an example, we discuss the constraints from the JLab/Hall A data on the dominant $u$-quark GPD $H$. The question arises whether GPDs are governed by some kind of holographic principle.
\end{abstract}

Keywords: generalized parton distributions, deeply virtual Compton scattering, sum rules, duality

PACS numbers: 11.30.Cp, 13.60.Fz, 11.30.Ly, 11.55.Hx 


\section{Contents}

1 Introduction

2 Mainstream GPD models versus experiment 5

2.1 GPD basics and terminology . . . . . . . . . . . . . . . . 5

2.1.1 Double distribution representation . . . . . . . . . . . . . . 5

2.1.2 Conformal partial wave expansions . . . . . . . . . . . . . . . 7

2.1.3 Other GPD expansions . . . . . . . . . . . . . . . . . . 8

2.2 Phenomenological applications . . . . . . . . . . . . . . . . . . . . 8

2.2.1 Ad hoc GPD phenomenology . . . . . . . . . . . . . . . . 9

2.2 .2 A fitting procedure . . . . . . . . . . . . . . 12

2.2.3 Lessons from failure and success: a speculative GPD picture . . . . . . . 13

3 GPD duality and families of GPD sum rules $\quad 15$

3.1 Preliminaries .......................... . . . 15

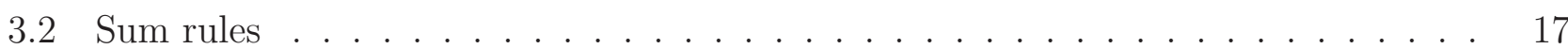

3.3 Deconvolution and duality ......................... 20 20

4 Phenomenological applications $\quad 24$

4.1 Strategy to access GPDs . . . . . . . . . . . . . . . . . . 2 24

4.2 Revealing GPDs from experimental measurements . . . . . . . . . . . . . . . 30

\begin{tabular}{ll|l|}
\hline & Outlook & 33 \\
\hline
\end{tabular} 


\section{Introduction}

Generalized parton distributions (GPDs) [1, 2, 3] are partonic amplitudes which contain the nonperturbative physics of perturbatively factorized exclusive leptoproduction amplitudes [4, 5]. Depending on the parton momentum fractions, a GPD is interpreted either as the probability amplitude of meson-like $t$-channel exchange or as $s$-channel exchange of a parton. Compared to parton distribution functions (PDFs) and distribution amplitudes, they reveal the partonic content of hadrons, protons in particular, from a complementary perspective; see for reviews [6, 7]. For instance, the partonic angular momentum, naturally defined by the decomposition of the angular momentum tensor in terms of quark and gluonic degrees of freedom, is given by the first Mellin moment of certain GPDs [8]. Quantification of such partonic decomposition of the proton spin is one of the main goals for measurements of GPD related processes.

During the previous decade, experimental effort has been devoted to the measurement of deep virtual Compton scattering (DVCS) [9, 10, 11, 12, 13, 14, 15, 16, 17, 18, 19, 20] and production of mesons, see e.g. Refs. [21, 22, 23, 24, 25], yielding an increasing amount and precision of experimental data. On the other hand, GPDs are intricate functions, depending on the parton's longitudinal momentum fraction $(x)$, the skewness $(\eta)$, the momentum transfer squared $(t)$, and the factorization scale ( $\mu^{2}$ - usually set equal to the virtuality $\mathcal{Q}^{2}$ of the probe). This makes their extraction from experimental measurements, where they enter as a convolution with a hardscattering amplitude, a rather awkward task. Since the partonic momentum fraction dependence of GPDs is integrated out in the amplitude, the degrees of freedom that can be constrained by experiment do not provide the momentum fraction shape of GPDs for fixed $\eta$. Consequently, GPD moments, including the angular momentum of quarks, cannot be directly revealed.

The analysis of deep virtual exclusive processes is based on GPD ansätze and models which take into account that GPDs reduce by sum rules to form factors and in the forward limit to PDFs. As the basic GPD ansatz, written in essence as a product of form factor and PDF, the popular VGG code [26] successfully describes the first DVCS beam spin asymmetry measurements at HERMES [10] and CLAS [11], but it fails in the case of more recent data on the unpolarized DVCS cross section, measured by the Hall A collaboration [14]. Other GPD models [27, 28, 29, 30] are similarly (un)successful in describing DVCS observables measured in fixed target experiments. In collider kinematics, no published GPD model, which is (a) mathematically consistent, (b) has a reasonable t-dependence, and (c) satisfies the evolution equation, is able to describe the DVCS data at leading order (LO) accuracy. Taking into account radiative corrections, the measured DVCS cross section can be described, as advocated in Refs. [31, 32], and fitted in Refs. [33, 34]. Also, Regge inspired modelling of off-shell amplitudes [35] can be tuned to be consistent with experimental data [36, 37]. If unitarity constraints are taken into account in the fixed target kinematics, the model [36] is in 
fair agreement with the unpolarized DVCS cross section measurement [38. Thereby, it provides a physical interpretation in terms of hadronic degrees of freedom, where the desired view inside the nucleon cannot be delivered.

Thus, although GPD modelling is guided by the relation of GPDs to PDFs and form factors, by Regge phenomenology, various model calculations, lattice simulations etc., the resulting models are only partially able to describe experimental data. This, in combination with the wide-spread implicit belief that the resulting GPD models might be realistic in a more general way, leads then to endless discussions, such as 'Which model is right, A or B?' (when both are maybe wrong) or 'GPDs or not GPDs?', to claims that GPDs can be constrained from present lattice data, and to 'a conjectured proof' of the breakdown of the GPD formalism (for some details see [39]). Sometimes, criticism of GPD models is unjustly extended to GPD representations. In our opinion it is unclear whether sufficient understanding of GPDs is reached in the proposed models. Below we shall demonstrate that there should be no problem to describe presently measured DVCS observables within the GPD formalism. Hence, we should question the present phenomenological approach, which is based on GPD models that we consider to be ad hoc constructions.

We would like to come back to two basic questions, which should be clearly answered.

- What GPD information can be revealed from experimental data?

- What do we learn from this information?

Both above questions have been addressed in the momentum fraction representation and, more recently, in the framework of the 'dual' GPD parameterization [40, 41, 30]. We shall recall the known partial answers to those questions in the momentum fraction representation and add a rarely recognized GPD aspect, namely, duality [42, 34], with the focus on the phenomenological applicability. Before we give the technicalities in the main body of the paper, we briefly outline the answers here. In our opinion it does not matter in which representation they are formulated, and in this paper we prefer the momentum fraction representation.

The answer to the first question arises from analyticity and it has been clearly spelled out for a fixed resolution scale in Ref. [43]. Let us recall that the DVCS amplitude is considered to be a holomorphic function, see, e.g., Refs. [44, 45, 43, 34, 46, 47], whose real part can be expressed in terms of its imaginary part, i.e., its $s$ - and $u$-channel discontinuities, via a single variable dispersion relation for fixed $t$. Moreover, to LO the imaginary part is given by the GPD on its cross-over trajectory $(\eta=x)$, which separates the meson-like $t$-channel exchange interpretation of the central region $(|x| \leq \eta)$ from the partonic $s$-channel exchange view of the outer region $(\eta \leq x \leq 1)$. Hence, apart from the cross-over trajectory the GPD for fixed resolution scale cannot be experimentally accessed. The dispersion relation gives us a considerable handle on the GPD at the cross-over 
trajectory and constrains its functional form, even if the modulus and phase of the amplitude are measured only in a restricted phase space. Therefore, the answer to the first question guides us to a simple phenomenological concept in which the dispersion relation is used to pin down the value of the GPD on the cross-over trajectory.

Suppose we know now the GPD at its cross-over trajectory from an (ideal) measurement for fixed photon virtuality $\mathcal{Q}^{2}$, as illustrated below in Fig. 1. The deconvolution problem, which then pops up, is how the GPD at $\eta=x$ is related to its value at any $\eta$, i.e., for the whole support. Lorentz covariance guarantees that a GPD possesses a duality property [42, 34], i.e., it can be mapped from the outer region into its central one, where the GPD is continuous on the crossover trajectory. Obviously, one can draw a priory any surface over the outer region of the GPD support that connects the curves given by the GPD at $\eta=x$ and $\eta=0$ for $0 \leq x \leq 1$, see Fig. 1. Positivity constraints, see Ref. [48, 49] and references therein, lead to constraints on these surfaces. The dual surfaces in the central region are then governed by Lorentz covariance. Each of these surfaces corresponds to a GPD model, and so the deconvolution problem has infinite solutions. The problem with present ad hoc GPD models is that usually one solution is picked out, with parameterization of the GPD at the cross-over trajectory being too rigid. We stress that evolution of GPD with change of the resolution scale provides a supplementary handle on the deconvolution problem, as advocated in Ref. [51]. However, for a small lever arm in the photon virtuality, only if some holographic principle exists, rigidly relating shape of this surface to values along the $\eta=x$ cross-over trajectory, the deconvolution problem can be strictly solved. But even if this hypothetical principle remains unknown, one still has various theoretical and phenomenological tools at hand to address the deconvolution problem and, in particular, the related problem of decomposition of the quark angular momentum.

In this paper we introduce a new set of such tools, namely GPD sum rules, and we illustrate how an alternative phenomenological approach to DVCS might look like. The outline is as follows. To shed light on unrealistic features of the GPD models, presently used in phenomenology, we give in Sect. 2 a short overview of experimental findings and their confrontation with GPD models, mainly for the DVCS process. The reader who is not interested in this phenomenological aspect can safely skip this section. In Sect. 3] we employ previous results [42, 34] to clearly spell out that GPDs have an internal duality, relating the outer and central regions. We derive then various GPD sum rules. In Sect. 4 we discuss the phenomenological applications of these sum rules and also give a preliminary example for fixed target kinematics. Finally, in Sect. 5] we summarize our findings and conjecture that GPDs should be governed by a holographic principle, which might be naturally related both to the duality interpretation of strong interaction phenomena, reviewed, e.g., in Ref. [52], and to the AdS/CFT conjecture [53, 54, 55]. 


\section{Mainstream GPD models versus experiment}

Numerous experimental, theoretical, and phenomenological efforts, reviewed in Refs. [6, 17, have been spent during the first decade of GPD phenomenology in an attempt to go beyond the basic GPD ansatz, given by the product of PDF and elastic form factor. Fortunately, there is tremendous progress on the experimental side and, certainly, some theoretical understanding is reached, too. Unfortunately, some rather hypothetical elements became an important part of the mainstream phenomenology, while at the same time some well-understood theoretical features are incompletely implemented. In what follows, we would like to make it clear that the observed phenomenological problem, namely, that certain GPD models fail to describe the DVCS data [14, 25, 56, 30], indicates only that the ad hoc GPD model approach in its present form is an inefficient tool for the future and that any general conclusion about the GPD formalism drawn from the failure of certain GPD models should be considered as a speculation. Below we briefly describe the situation and attempt to separate different problems, which are often mixed up.

\subsection{GPD basics and terminology}

GPD models, used in phenomenology so far, are mainly based either on a spectral representation for the Green functions of light-ray operators, so-called double distributions (DDs) [1, 57], or on the collinear conformal partial wave expansion1 [59], known in various versions [60, 61, 62, 63, 42, 64, 65]. Since they all by definition represent the same field theoretical object, any GPD model, as long as it respects basic properties, such as Lorentz covariance, can be represented in any of the named representations. Another popular GPD representation is given as overlap of light-cone wave functions [66, 67, 68. We emphasize that the one-to-one correspondence of the DD and overlap light-cone wave function representation [66, 67, 68, should hold on general field theoretical grounds, too. This has been demonstrated within wave function models in Refs. [69, 70, 71]. In the overlap light-cone wave function representation the positivity constraints [48, 49] are explicitly satisfied 2 These constraints are exactly valid at LO approximation; however, their power drastically diminishes if one calculates the physical amplitude.

\subsubsection{Double distribution representation}

The DD representation arises directly from diagrammatical considerations [1, 57]. We consider it to be the most general representation that inherits properties of field theory. Here, the partonic

\footnotetext{
${ }^{1}$ The group theoretical aspects and QCD applications of the collinear conformal group are given in Ref. [58].

2 The solution of these general positivity constraints can be interpreted as a two body light-cone wave function overlap representation for a collective spectator [50].
} 
quantum numbers are the momentum fractions of two partons. We recall that the first sophisticated GPD ansatz was elaborated in Refs. [72, 73] for $t=0$ via a DD ansatz, which factorizes in a PDF and a single variable profile function whose shape, viewed as a meson distribution amplitude, was assumed to be convex. For brevity, we call this the Radyushkin's DD ansatz (RDDA). Because of its convenient parameterization, it was adopted by many model builders in various $t$-decorated versions and within generalizations of the profile function; however, the convexity of its shape was not touched upon. In particular, the RDDA [72, 73] has been utilized in Ref. [74] to build up a GPD model, guided by the results of the chiral quark soliton model ( $\chi \mathrm{QSM})$, decorated with a factorized or Regge inspired $t$-dependence, and based on certain PDF parameterizations. This GPD model is now numerically implemented in the popular VGG code [26]. Here a descendant of the $D$-term has been utilized to parameterize the GPD $E$ in terms of the quark angular momentum. We follow the common terminology and call this code the VGG model. We note that VGG results might be different from other GPD models that were written down in a similar fashion within RDDA, e.g., in Ref. [27].

It was already emphasized in Refs. [72, 73, 75] that more realistic GPD models should possess an intricate interplay of momentum fraction and momentum transfer squared dependence. It turned out that guidance for a more realistic $t$-dependence comes from field theory inspired models. Such an improvement is now implemented in GPD models for the pion [69] and nucleon [70, 71]. All these investigations started from overlap representations with power-law wave functions, and, aiming at different goals, they finally provided rather analogous expressions for the resulting DDs 3 Such models can also be obtained from the evaluation of (general) triangle Feynman diagrams. To emphasize the physical content of these GPD models, one might also denote them 'collective spectator quark models' 4 For both $\eta=0$ and $\eta=x$ the $t$-dependence of these models dies out in the limit $x \rightarrow 1$; see, e.g., discussion in Ref. [71]. The behavior in the former case coincides with lattice measurements of generalized form factors [77, 78, 79, 80, 81, 82, 83]. A more general DD ansatz in the form of a factorized RDDA with the common improvement of Regge behavior has been written down for certain proton GPDs in Ref. [71]; for the pion case see Ref. [69]. We have also convinced ourselves that one should not insist on a convex shape of the profile function, and could, e.g., add 'higher' modes, to get rather flexible GPD models. In this way one can replace

\footnotetext{
${ }^{3}$ It was observed in Ref. 69 that the functional form of light-cone wave functions cannot be arbitrarily chosen to derive the DD representation. Therefore, the authors of Ref. [70] started with a covariant quark model to obtain GPD models, formulated in DD representation. In Ref. [71 it was stated that in the light-cone wave function overlap representation the longitudinal and transversal degrees of freedom are tied to each other due to Lorentz symmetry. Hence, arbitrary modelling of light-cone wave functions will usually violate the polynomiality property of GPD moments, see, e.g., Ref. [76].

${ }^{4}$ There is a huge literature in which GPDs have been studied within dynamical model approaches. The reader may find references in Refs. 6, 71, 76].
} 
ad hoc GPD model approach with a more flexible fitting procedure.

\subsubsection{Conformal partial wave expansions}

The expansion of the GPD in collinear $S L(2, \mathbb{R})$ conformal partial waves arises naturally from the solution of the evolution equation to LO accuracy. The parameterizations, applied in phenomenology, are named 'dual' [84] or Mellin-Barnes representation [42]. In our understanding, the name 'dual' primarily refers to the use of crossing symmetry [1, 85, 86], which is, by itself, independent of the chosen representation. For the sake of clarity, we follow the authors of Ref. [84] and denote their representation 'dual' in quotation marks. The term dual (without quotation marks) we use for a general GPD property, as used above and explained further in Sect. 3, This concept was partially utilized for evolution kernels [87, 1] and has been adopted to GPDs [42, supplemented by a constructive proof [34]. As emphasized in Ref. [42] it is also tied to crossing, again independently of the representation. The term Mellin-Barnes representation refers to a specific integral representation of the collinear conformal partial wave expansion, closely related to the well-known Sommerfeld-Watson transform, in which internal GPD duality is manifestly incorporated, cf. Ref. [64, 65].

In the Mellin-Barnes representation, besides the complex-valued conformal spin, the angular momentum of the $t$-channel $\mathrm{SO}(3)$ partial wave might be employed as a label. In the 'dual' parameterization a forward-like momentum fraction (conjugated variable to the conformal spin) and the difference of integral conformal spin and $t$-channel angular momentum appear as labels. This makes contact to hadronic physics [84], in particular to Regge phenomenology [34]. In the minimalist version of this model the expansion of conformal moments in terms of $\mathrm{SO}(3) t$-channel partial waves is restricted to the leading one. The inclusion of the next-leading $\mathrm{SO}(3)$ partial wave has been called minimal 'dual' parameterization [84]. More recently it has become clear how the inclusion of non-leading $\mathrm{SO}(3)$ partial waves can be effectively achieved in the 'dual' parameterization [41. The minimalist and minimal version in the 'dual' parameterization corresponds to the leading and next-leading $\mathrm{SO}(3)$ partial wave approximation. We use the terminology: leading (i.e., minimalist) $\mathrm{SO}(3)$ partial wave and next-leading (i.e., minimal) $\mathrm{SO}(3)$ partial wave approximation 34. GPD modelling in the 'dual' and the Mellin-Barnes integral parameterizations is presently done with different phenomenological emphases. In the former parameterization the model aspect with respect to the approximation of $\mathrm{SO}(3)$ partial waves, see Sect. 4.1, is pronounced [40, 41, while in the latter, up to now applied only to small $x_{\mathrm{Bj}}$ physics, the Regge aspect [34] and the resummation of $\mathrm{SO}(3)$ partial waves [88] is given priority. 

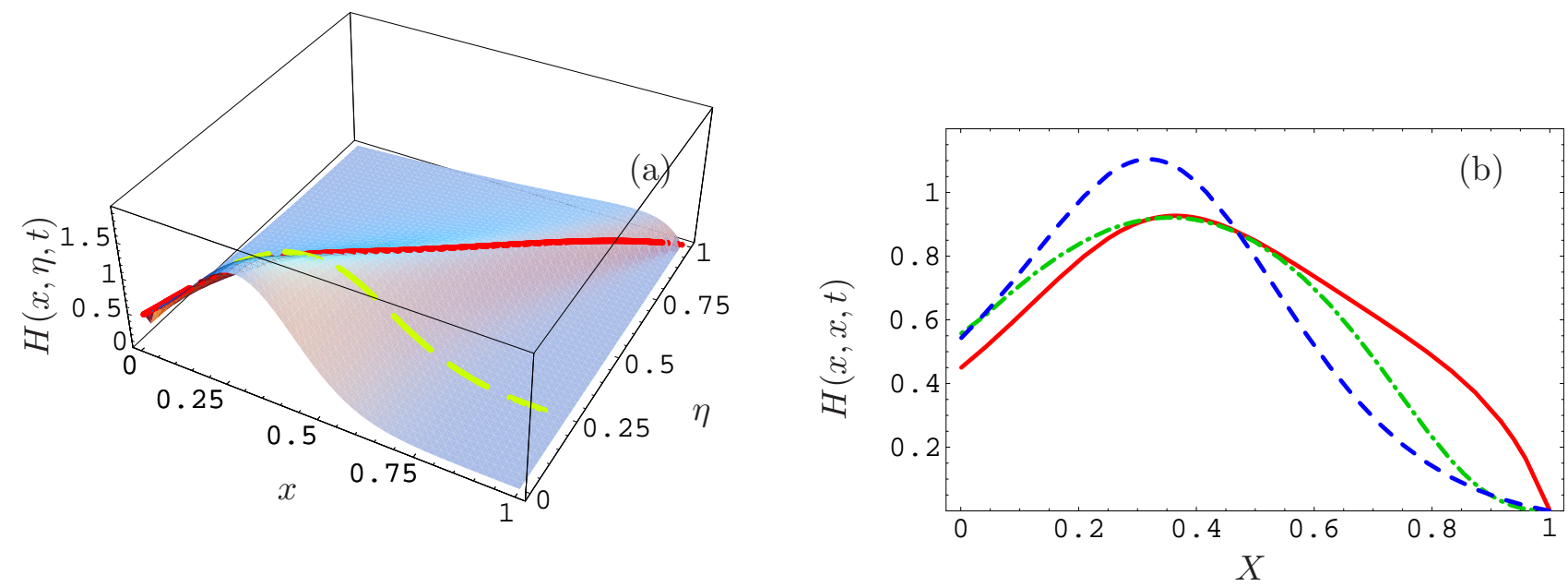

Figure 1: The left panel displays the spectator model of Ref. [71, as specified there in Eq. (25), with $t=t_{\min }-0.25 \mathrm{GeV}^{2}$. The red solid curve visualizes the experimentally accessible information for fixed photon virtuality, i.e., the GPD at the cross-over trajectory $\eta=x$, while the dashed one is an example curve that can be used to evaluate the real part of CFFs via Eq. (4). In the right panel the same GPD at $\eta=x$ (solid) is displayed versus the Bjorken-like variable $X=2 x /(1+x)$ and it is compared with a VGG-like (dashed) and the leading $\mathrm{SO}(3)$ partial wave approximation of the Mellin-Barnes (dash-dotted) ansatz. Both ansätze are based on the zero skewness spectator GPD model and possess a factorized $t$-dependence.

\subsubsection{Other GPD expansions}

One might also introduce an expansion with respect to a complete basis of orthogonal polynomials, such as Gegenbauer [59] or Bernstein [89].5 Such expansions can in principle be expressed in the two considered ones. The expansion in terms of Bernstein polynomials has been proposed once in deep-inelastic scattering (DIS) [90; however, it could not compete with the standard approaches, employed in global DIS fits. Also, we know from experience that the expansion of GPDs with respect to a complete basis of orthogonal polynomials turns out to be numerically inefficient at smaller values of $x_{\mathrm{Bj}} 92$.

\subsection{Phenomenological applications}

The phenomenological application of GPDs for the deep virtual leptoproduction of mesons or photon is based on factorization theorems, proven to leading order in the $1 / \mathcal{Q}$ expansion [4, 5].

\footnotetext{
${ }^{5}$ Interestingly, in Ref. 91] the authors started from a covariantly formulated spectator model; however, consider Lorentz covariance (polynomiality of GPD moments) as a DD hypothesis. Finally, they are doing GPD modelling for $\eta=0$. To include skewness dependence, they parameterize the GPD in terms of a few Bernstein polynomials and rely on lattice data [89].
} 
Thereby it is assumed that GPDs are continuous at the cross-over point. It is commonly assumed that the GPD framework is quantitatively applicable for DVCS at a scale of the order of one or few $\mathrm{GeV}^{2}$, while for meson production it might be of the order of $10 \mathrm{GeV}^{2}$. Unfortunately, only very little is known about the size of power suppressed contributions and it remains an open problem to pin down the onset scale for the GPD formalism. This problem might also be addressed from the phenomenological side. In the following we assume that the GPD formalism is quantitatively applicable to leading order of the perturbative expansion in the QCD coupling constant for DVCS measurements in JLAB, HERMES and H1/ZEUS kinematics, where the photon virtuality is larger than $1 \mathrm{GeV}^{2}$.

The specific GPD models are all more or less constrained by the same phenomenological input, PDFs, form factors, and sometimes model or lattice estimates. Roughly spoken, the difference in the physical content of various GPD models at $\eta=x$ is more or less accidental and arises from simplifications, mainly done for convenience, in a given representation. Obviously, the model dependence is still visible in the described deep virtual amplitudes; however, the parameterization thereof is effectively not under control. This is illustrated in the right panel of Fig. 1, where we show the GPD at its cross-over trajectory within the spectator model (solid) of Ref. [71], the leading $\mathrm{SO}(3)$ partial wave approximation in the Mellin-Barnes representation (dash-dotted), and a VGG-like ansatz (dashed). For the latter two models we have used a factorized $t$-dependence, and the form factor and PDF were evaluated in the spectator model. There is one rule which GPD model builders like to follow; namely, they mostly avoid invisible degrees of freedom (those that die out in the forward limit). In other words, one relies on the implicit assumption that the holographic principle holds true. Exceptions to this rule are the pion pole contribution [93, 74] and the so-called $D$-term [94], where the latter is entirely related to the subtraction constant in the dispersion relation [43]. In the $\mathrm{SO}(3) t$-channel partial wave expansion both of these exceptions are assigned to $J=0$ contributions, as shown for the subtraction constant in Sect. 4. In the context of the parton model [95], and within its field theoretical model realization for forward Compton scattering [96], such a $J=0$ term relates low- and high-energy physics. We emphasize that there are various possibilities to embody these $J=0$ terms into GPD models, see, e.g., Refs. [94, 97, 86, 70, 34, 71], and they might lead to rather different ad hoc GPD 'predictions'.

\subsubsection{Ad hoc GPD phenomenology}

The qualitative failure of the VGG model in describing virtual electroproduction of $\rho^{0}$ mesons in the resonance, i.e., large $x_{\mathrm{Bj}}$, region [25] is partly related to its ad hoc $t$-dependence. Certainly, here one has to be concerned about the applicability of perturbative QCD (and, by the way, also about the onset of the Regge regime) and so one might expect in this region both large radiative and 
power suppressed corrections. However, since the Feynman mechanism is somehow incorporated in the GPD formalism, we might expect that the $t$-dependence of the hadronic amplitude is qualitatively embodied in the GPD; see Ref. [98, 99] for a perhaps more appropriate realization of the GPD formalism in this kinematics. The $t$-dependence of the cross section diminishes in the large $x_{\mathrm{Bj}}$ region [25], which is consistent with the aforementioned spectator GPD model [71] 6] The notorious normalization problem of the cross section in this kinematical region is to LO approximation entirely related to the end-point behavior of the VGG model. It was cured in Ref. [25] by a $D$-term inspired addendum, concentrated in the central GPD region and vanishing in the forward kinematics. We will also illustrate in Sect. 4.2 that one can easily construct GPD ansätze which could fix the normalization problem in a different manner. In fact, it is demonstrated in Fig. 1(b), that a simple spectator model (solid curve) possesses in comparison to a VGG-like ansatz (dashed curve) an enhancement effect in the large momentum fraction region.

The DVCS amplitude is accessible in the deep virtual electroproduction of photons, where due to the interference of the bremsstrahlung and DVCS processes one can also reveal the phase of the DVCS amplitude. The VGG mode 7 also fails to describe the beam spin asymmetry and polarized cross section for unpolarized proton in the fixed target JLab kinematics, see e.g., Ref. [14], since here it does not offer the possibility to decrease the normalization of the resulting amplitudes, compared to the basic GPD ansatz.

The normalization problem was already studied in Refs. [27, 28] and to some extent cured by reducing the amount of sea quark contributions (introducing so-called model A or B, which, however, have an unrealistic $t$-dependence). In fact, adjusting the normalization of the DVCS amplitude by suppression of sea quark contribution 8 makes it possible to describe existing DVCS data, related to beam spin asymmetries or polarized cross section measurements. Even semiquantitative discussions [27, 28] seem to provide empirical understanding, when employed within

\footnotetext{
${ }^{6}$ We thank the authors of Ref. [25, asking the question "GPDs or not GPDs?", for clarification. Their conclusions about GPD models within DD representation refer only to specific models, considered there. In particular the statement "the very flat $t$ slopes observed can only arise from GPD contributions not constrained by the FF [form factor] sum rule" is not meant to be valid in general. Indeed, simple spectator GPD models, formulated in DD representation and satisfying the form factor sum rule, explain the observation.

7 We would like to emphasize that the 'easy to tune' options in GPD codes indirectly suggest the possibility of easy phenomenological GPD interpretation; however, we fear that this can be misleading. For instance, it was found in Ref. 20 that the D-term within the VGG model is disfavored from the HERMES DVCS measurements. Since the $\chi$ QSM [74, 100] and now also lattice calculations, e.g., in Ref. [83], indicate that the $D$-term is sizeable, our interpretation of this statement is that the VGG model, the incorporation of the $D$-term equivalent part (see Sect. 3.3) in the model or a combination of both, is improper. Also, the model dependent constraints on the quark angular momentum in Ref. 20] illustrate that the model dependence is not under control.

${ }^{8}$ Sea quark contributions and in our understanding also valence singlet quark ones are neglected in the DVCS results of Ref. [89.
} 
the impulse approximation in a prediction of beam spin asymmetry for DVCS on nuclei [101. If this 'prediction' turns out to be in disagreement with increasingly precise experimental data, it will be exciting to employ the GPD formalism as a tool to explore the interplay of nucleon and partonic degrees of freedom beyond the impulse approximation.

The minimal(ist) version of the 'dual' parameterization [84] has been confronted with DVCS data in Refs. [29] and [30] and describes asymmetries and polarized cross sections. Finally, all these GPD models fail to describe the unpolarized DVCS cross section, measured by the Hall A collaboration at JLab [14].

The understanding of GPDs in collider kinematics of the H1 and ZEUS collaborations [9, 12, 13, 18, was in poor state, too. Claims that the DVCS cross section can be described within published GPD models to LO accuracy are based on either a problematic implementation of the $t$ dependence [27, 29], 9 violation of the perturbatively predicted scale change [29, 102, 10, or violation of basic GPD properties [102] 11

We stress that the physical content of the LO normalization problem was known to the authors of Ref. [102. Although they started to analyze the model problems within the RDDA, they finally gave up on the DD representation. Armed with the aligned jet model, a reasonable physical picture, however, violating Lorentz covariance, they came up with an inconsistent GPD model (see footnote 11), which must be rejected. After restoring Lorentz covariance in their model one simply finds a PDF which is decorated with $t$-dependence - a special case of the RDDA.

The normalization problem can be naturally overcome by inclusion of radiative corrections as advocated in Refs. [31, 32]. However, it was at the same time observed that GPD ansätze within different PDF parameterizations, describing the same set of unpolarized DIS data, result in rather different DVCS predictions [92]. This is quite understandable if one remembers that already the description of the unpolarized DIS structure functions is a fine tuning problem. There the perturbative expansion of the evolution is unstable in the small $x_{\mathrm{Bj}}$ region, see discussion in Ref. [103], and the same thing now happens in the DVCS case [34]. We emphasize that this does not disqualify the perturbative approach to DIS and DVCS at small $x_{\mathrm{Bj}}$, because the evolution operator is universal, i.e., process independent. Also a resummation procedure might

\footnotetext{
${ }^{9}$ Since the $t$-dependence of the sea quark GPD is unrealistic, we rejected the model of Ref. [27 for some time. However, it describes various DVCS data and we employ it for error estimates in Sect. 4.2. The description of data in Ref. 29] arises from a GPD model that induces an unrealistic shrinkage effect of the diffractive forward peak.

10 The incorporation of the $\mathcal{Q}^{2}$-dependent $t$-slope (32) in Ref. [102, also adopted in Ref. [29, violates the LO scale change prediction.

11 The aligned jet GPD model 102 is based on a truncated Taylor expansion in $\eta$ for all GPD moments. The inconsistency of the proposal [102] was revealed in Ref. [46]. According to Eq. (21), the moments in the 'full' model, evaluated from Eq. (21, [102]), are $\eta$-independent and the resulting GPD is the PDF decorated with a $t$-dependence.
} 

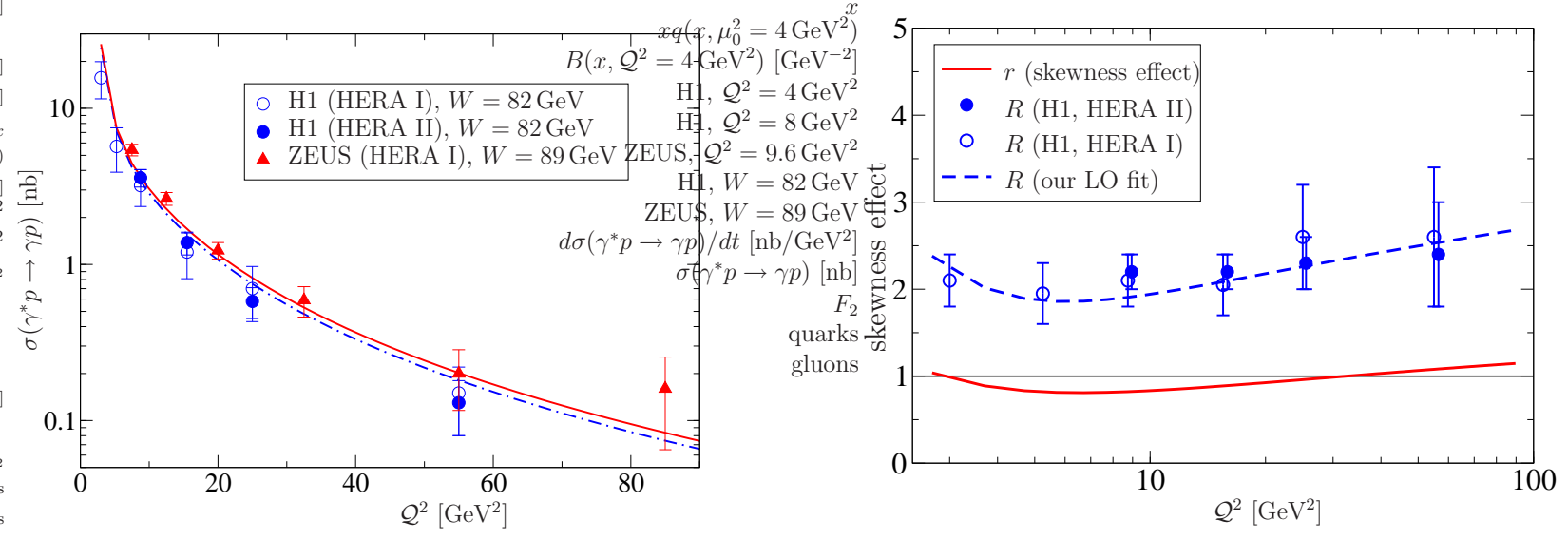

Figure 2: The left panel shows a preliminary LO fit result [88] to the HERA DVCS data set versus the photon virtuality $\mathcal{Q}^{2}$ : ZEUS [12] with $W=89 \mathrm{GeV}$ (triangle, solid curve) and H1 with $W=$ $82 \mathrm{GeV}$ within the HERA I [13] (empty circle, dash-dotted curve) and HERA II [18] (full circle, dash-dotted curve) run. In the right panel we show the skewness ratio $R$, defined in Ref. [18] as observable, (dashed curve) to LO approximation, i.e., $R \approx H\left(x, x, t=0, \mathcal{Q}^{2}\right) / H\left(2 x, 0, t=0, \mathcal{Q}^{2}\right)$ for fixed $W=82 \mathrm{GeV}$, and the ratio $r \equiv H\left(x, x, t=0, \mathcal{Q}^{2}\right) / H\left(x, 0, t=0, \mathcal{Q}^{2}\right)$ for fixed $x=10^{-3}$ (solid curve). Note that $R \approx 2^{\alpha\left(0, \mathcal{Q}^{2}\right)} r$ with $\alpha\left(0, \mathcal{Q}^{2} \sim 4 \mathrm{GeV}^{2}\right) \sim 1.2$.

stabilize the perturbative results; for a discussion see, e.g., Ref. [104]. In particular, it will be phenomenologically important for a perturbative GPD analysis of the deep virtual vector meson electroproduction, see Ref. [105] for details.

\subsubsection{A fitting procedure}

The ad hoc GPD ansatz approach is surely not a proper framework for revealing the partonic content of the proton. Insight in the perturbative GPD approach is needed, too. We started to explore an alternative framework for DVCS in the small $x_{\text {Bj }}$ kinematics [106, 34, 88, up to next-to-next-to-leading order [107, 108, 109, 110, 33] and would like here to present what we have learned. Since radiative corrections to the DVCS amplitude (or DIS structure function $F_{T}$ ) can be essentially absorbed by a scheme change [27], the description of experimental DVCS data at LO accuracy is the indispensable key to a GPD understanding.

In the small $x_{\mathrm{Bj}}$ kinematics the functional form of the amplitude simplifies drastically and the reggeized $t$-channel view gives guidance for modelling. This is a pure empirical recipe, where one has to replace the classical pomeron trajectory by an effective one. The effective pomeron intercept is an integral part of the universal GPD and it is considered to be skewness independent, i.e., the GPDs or DVCS amplitude have the same intercept as the corresponding PDFs or DIS structure function, respectively. This is supported by both a diagrammatical $t$-channel ladder analysis [44] 
and the experimental data.

For a fitting routine it is crucial to have full control over the normalization of the DVCS amplitude. This can be achieved within invisible terms that die out in forward kinematics. The use of conformal symmetry enabled us to study the GPD approach up to next-to-next-to-leading order in perturbation theory [110, 33, 34]. The numerical advantages of the Mellin-Barnes representation then allow for a simultaneous fitting procedure of DVCS and DIS data. The essential lesson from our GPD fits is that a leading $\mathrm{SO}(3)$ partial wave approximation (minimalist 'dual' model) does not work in the small $x_{\mathrm{Bj}}$ regime at LO, but only at perturbative next-to-leading order or beyond [34]. Within the conformal partial wave expansion, a more flexible parameterization of GPDs has been worked out by several researchers; however, it is only partly published [111, 112, 40, 88, After such an improvement, the experimental data for collider kinematics can be fitted at LO accuracy either within the inclusion of the next-leading $\mathrm{SO}(3)$ partial wave [111] or a flexible model dependent $\mathrm{SO}(3)$ partial wave resummation [88]. A preliminary fitting of results to the DVCS cross section, measured by the H1 [13, 18] and ZEUS [12] collaborations, versus the photon virtuality $\mathcal{Q}^{2}$ [88] is displayed in the left panel of Fig. 2. Our result is that the dominant sea quark GPD at low $x$ shows to LO accuracy almost no skewness effect [88]; see the solid curve in the right panel of Fig. 2. We should here give attention to a different GPD interpretation of the same DVCS data. Namely, the measured ratio $R$ [18] and our corresponding GPD fit (dashed line), might be viewed as a large skewness effect. Actually, using a better suited $r$ function, we reinterpret this as (almost) no-skewness effect. Such a vanishing skewness effect was for a lower resolution scale advocated in Ref. [44, but it turns out that it holds for the experimental lever arm of $\sim 3 \cdots 80 \mathrm{GeV}^{2}$ [18]. In the published GPD models this no-skewness property was either never present or, if it was taken at low input scale [44], the ansatz spoils it due to the evolution [44]. Unfortunately, the large evolution effect was propagated as a 'prediction' [113], it is 'naturally' implemented in approximated versions of conformal partial wave GPD representations, and it occurs in a weaker form for RDDA, see Ref. [114]. We add that in the phenomenology of diffractive vector meson production it is often taken for granted that the GPDs possess a positive non-zero skewness effect, e.g., in Ref. [115].

\subsubsection{Lessons from failure and success: a speculative GPD picture}

From a phenomenological failure of present GPD models one can mainly conclude that its ad hoc realization was improper. It is senseless to criticize GPD representations in general, which will just lead to a step backwards. GPD features which have been understood in one representation can be incorporated in the other representation, too. Furthermore, from the confrontation of experimental findings and present GPD models one cannot provide a reliable partonic interpreta- 
tion, in particular for the $D$-term equivalent part and the quark angular momentum. The critique of the perturbative approach offered so far from the outcome of such a confrontation should be considered as speculation. In particular, from what was said above for DVCS, it follows that a perturbative GPD analysis for deeply virtual vector meson electroproduction at small $x_{\mathrm{Bj}}$ also has to be reconsidered and extended to next-to-leading order.

The failure of the mainstream ad hoc GPD models should be an incentive to learn the lessons and find improved versions. This has been achieved to some extent with respect to the interplay of momentum fraction and $t$-dependence [69, 70, 71]. Pragmatical solutions to fit data within the GPD formalism are obvious, namely, one has to exploit degrees of freedom that are invisible in the zero skewness case, e.g., more flexible profile functions. This sounds simple; however, it requires some technical effort and, more importantly, GPDs are intricate functions and so one can easily get 'lost'. Even in the in some sense much simpler case of small $x_{\text {Bj }}$ kinematics, three different groups of authors attempted in a period of five years or so to go for a GPD interpretation of the ZEUS/H1 DVCS data, where two of them gave up basic (and realistic) GPD properties. In our opinion the message from that for the analysis of fixed target experiments is that one should first utilize dispersion relation techniques, to pin down the GPD on its cross-over trajectory.

Nevertheless, we attempt in the following to catch the possible qualitative features of a "more realistic' GPD at its cross-over trajectory from the phenomenological findings and we would like to share our speculations on how it might look, away from the cross-over trajectory. These unorthodox considerations are disputable and we consider the outcome only as a working hypothesis.

Let us briefly summarize the phenomenological findings. Present ad hoc GPD models, perhaps apart from the VGG one, are in the valence region consistent with (beam) spin asymmetry and polarized cross section measurements. If they are tuned to experimental data they might possess some kind of predictive power for certain observables. There is now a clear understanding of the dominant sea quark GPD in the small $x_{\mathrm{Bj}}$ region, namely, the skewness effect to LO accuracy is compatible with zero. The extension of such an ansatz to larger values of $x$ induces a wrong scale dependence. The large size of the unpolarized DVCS cross section in the valence region is hardly understandable within present GPD models. The virtual $\rho^{0}$ electroproduction data [25] in the large $x_{\mathrm{Bj}}$ region are consistent with respect to the $t$-dependence of simple spectator models. A precise quantitative understanding of the cross section is for various reasons rather challenging. We add that the employment of unitarity constraints in a Regge inspired model analysis [38] indicates that for DVCS already the valence region cannot be correctly described with a few reggeized $t$-channel exchanges.

Since the real part of the DVCS amplitude can be determined from a dispersion relation, a GPD ansatz at the cross-over trajectory must simultaneously give the correct description for 
the imaginary part of the amplitude in the whole phase space. This is certainly not the case for present GPD models; however, this GPD aspect can be straightforwardly analyzed using the growing amount of DVCS data.

On qualitative grounds we conclude from the experimental findings that a GPD on its crossover trajectory is at small $x$ governed by the reggeized $t$-channel view, where the skewness effect might be zero, while at large $x$ there is a large skewness effect and the $t$-dependence diminishes. Such a behavior is supported by field theory inspired spectator models [69, 70, 71]; see, e.g., the discussion in Ref. [71], where, however, the Regge behavior at small $x$ is absent. Implementing Regge-behavior from the $s$-channel view, along the lines of Ref. [116], yields at $t=0$ a Regge 'improved' RDDA that possesses an experimentally unfavored large skewness effect at small $x$. To find a more realistic behavior it is perhaps more appropriate to have the $t$-channel view by considering the diagrammatical ladder, as started in Ref. [44]. That this is a reliable concept has been demonstrated for PDFs, see, e.g., Ref. [117].

We finally mention that due to the evolution the GPD value in the outer region is reduced and 'flows' into the central region to 'fill' it, i.e., yielding a growing value of the GPD. From the experimental observation that the skewness effect at very small $x$ 'generically' does not depend on the resolution scale, one might draw a speculative conclusion. Namely, loosely spoken, the 'flow' into the central region has already stopped and the GPD in this region is mostly 'filled' at a resolution scale of about $2 \mathrm{GeV}$ or perhaps even less. This seems to fit the qualitative experimental findings at large $x_{\mathrm{Bj}}$, indicating a 'big' GPD contribution in the central region [25]. Utilizing the evolution equation, this pictorial speculation can be exactly formulated as a mathematical problem; however, this is beyond the scope of the paper.

\section{GPD duality and families of GPD sum rules}

In this section we deliver the tools that allow for a straightforward GPD analysis of the photon electroproduction data in fixed target kinematics. Thereby, we adopt well-known techniques, utilized in the phenomenology of on-shell processes and combine them with the duality property of GPDs [42, 34.

\subsection{Preliminaries}

To address the problems, we consider virtual Compton scattering off a proton in the generalized Bjorken region [1]:

$$
\gamma^{*}\left(q_{1}\right) p\left(p_{1}\right) \rightarrow \gamma^{(*)}\left(q_{2}\right) p\left(p_{2}\right), \quad Q^{2}=-\left(q_{1}+q_{2}\right)^{2} / 4 \rightarrow \infty
$$


where the momentum transfer squared $t \equiv \Delta^{2}=\left(p_{2}-p_{1}\right)^{2}$ and the two scaling variables

$$
\xi=\frac{Q^{2}}{P \cdot q}, \quad \eta=-\frac{\Delta \cdot q}{P \cdot q},
$$

are fixed. Here $P=p_{1}+p_{2}, \Delta=p_{2}-p_{1}$ and $q=\left(q_{1}+q_{2}\right) / 2$. We shall always take the values of $\xi$ and $\eta$ to be positive. In the twist-two approximation the crossing-invariant (i.e., for $s \leftrightarrow t$ ) photon asymmetry parameter is given by their ratio:

$$
\vartheta=\frac{q_{1}^{2}-q_{2}^{2}}{q_{1}^{2}+q_{2}^{2}} \simeq \frac{\eta}{\xi}
$$

The amplitude is parameterized in terms of the Compton form factors (CFFs), which in perturbative LO, Born or hand-bag approximation, read for leading twist-two:

$$
\mathcal{F}\left(\xi, \vartheta, t, Q^{2}\right) \stackrel{\mathrm{LO}}{=} \int_{-1}^{1} d x\left(\frac{1}{\xi-x-i \epsilon} \mp \frac{1}{\xi+x-i \epsilon}\right) F\left(x, \eta=\vartheta \xi, t, \mu^{2}=Q^{2}\right) .
$$

Here the scale $\mu^{2}$ is a factorization scale at which the hard-scattering part and GPDs are factorized and it is equated to the characteristic scale $Q^{2}$ of the process (the common choice). The explicit dependence of the hard-scattering part on $\mu^{2}$ appears at next-to-leading order. In our shorthand notation CFFs will be denoted $\mathcal{F}$ and the GPDs $F$, cf. Ref. [27]:

$$
\mathcal{F}=\left\{\begin{array}{c}
\mathcal{H}, \mathcal{E} \\
\widetilde{\mathcal{H}}, \widetilde{\mathcal{E}}
\end{array}\right\}\left(\xi, \vartheta, t, Q^{2}\right), \quad F=\left\{\begin{array}{c}
H, E \\
\widetilde{H}, \widetilde{E}
\end{array}\right\}\left(x, \eta=\vartheta \xi, t, \mu^{2}\right) \quad \text { for parity } \quad\left\{\begin{array}{c}
\text { even } \\
\text { odd }
\end{array}\right\} .
$$

The sign $\mp$ on the r.h.s. of Eq. (4) refers to the symmetry with respect to $x$. This convention will be consistently used below without an explicit reference to it. Note that antisymmetry (symmetry) with respect to $x$ corresponds to parity even (odd) CFFs, which also have signature $+1(-1)$. This definite signature originates from symmetry under the exchange of $s$ - and $u$-channel. Here we did not indicate fractional charge squared factors, which can easily be restored by replacing $F$ with $\sum_{a=u, d, \cdots} Q_{a}^{2}{ }^{a} F$. The GPDs $F\left(x, \eta, t, Q^{2}\right)$ are real-valued functions that are symmetric in $\eta[118$, and their $j$ th Mellin moments are polynomials in $\eta$ of order $j+1$ (polynomiality property). To be on safer ground concerning the analyticity hypothesis, we consider in the following only the Euclidean region, i.e., $|\vartheta| \leq 1$. This is ensured by the condition $\xi>\eta$. Unfortunately, apart from the contribution to virtual two-photon exchange corrections [119], the CFFs are only accessible in DIS $(\vartheta=0$, i.e., $\eta=0)$ and $\operatorname{DVCS}(\vartheta=1$, i.e., $\eta=\xi)$. In the former case we extract PDFs, equal to GPDs with $\eta=0$ and $t=0$, and in the latter case we find the GPDs on the cross-over trajectory $x=\eta$. For pedagogical reasons, however, we shall first do a gedanken experiment and suppose that the Compton amplitude can be 'measured' in the whole Euclidean region.

The obvious part of the answer to the question 'What GPD information can be revealed from experiment?' is that the imaginary part of the CFF (44) is given by the GPD combination:

$$
F^{\mp}\left(x, \eta, t, Q^{2}\right) \equiv F\left(x, \eta, t, Q^{2}\right) \mp F\left(-x, \eta, t, Q^{2}\right) \stackrel{\mathrm{LO}}{=} \frac{1}{\pi} \Im \mathrm{m} \mathcal{F}\left(\xi=x, \vartheta=\eta / x, t, Q^{2}\right) .
$$


This formula tells us that by varying the photon asymmetry parameter, i.e., $0 \leq \vartheta \leq 1$, the GPD is scanned in the outer region

$$
0 \leq \eta \leq x=\xi \leq 1 .
$$

This is analogous to DIS, where the structure functions are to LO equal to PDFs. The r.h.s. in Eq. (6) and so the GPD $F^{\mp}\left(x, \eta, t, Q^{2}\right)$ with $\eta \leq x$ might be viewed as the spectral function for this Compton scattering process, given by the $s$-channel cut. Hence, the outer region of the GPD $F\left(x, \eta, t, Q^{2}\right)$ with $\eta \leq x$ is expressed by the difference or sum of $s$ - and $u$-channel cuts. In accordance with the standard definition of the PDFs, the GPD (6) is understood for $\eta \leq x$ as the combination $F^{\mp}(x \geq \eta, \eta, \cdots)=\left[{ }^{q} F+{ }^{\bar{q}} F\right](x, \eta, \cdots)$ of quarks and antiquarks, where

$$
\left\{\begin{array}{l}
q F \\
\bar{q} F
\end{array}\right\}(x, \eta, \cdots)=\left\{\begin{array}{c}
F(x, \eta, \cdots) \\
\mp F(-x, \eta, \cdots)
\end{array}\right\} \quad\left\{\begin{array}{c}
\text { quark } \\
\text { antiquark }
\end{array} \text { for } x \geq \eta .\right.
$$

The definite symmetry of $F^{\mp}$ GPDs under reflection $x \rightarrow-x$ allows us to extend them uniquely to negative values $x \leq-\eta$. The support extension into the central region $x \in[-\eta, \eta]$ should be done in such a way that the polynomiality condition is satisfied. As we shall see in Sect. 3.3 below, analyticity guarantees that this procedure is unique.

\subsection{Sum rules}

To get deeper into the problem, we consider the CFFs as holomorphic functions of Mandelstam variables which satisfy the Schwartz reflection principle. Furthermore, we assume that for fixed (negative) value of $t$ in the Euclidean region the CFFs possess only elastic poles, as well as $s$ and $u$-channel discontinuities on the real axis. Hence, CFFs satisfy a single variable dispersion relation, where $t$ and the photon virtualities are considered as fixed variables; see, e.g., Refs. [44, 45, 43, 34, 46. Taking into account that they have a definite signature, this can be equivalently rewritten in terms of the crossing-symmetric variable $\nu=(s-u) / 4 M$, related to the energy of the initial photon, or in terms of the scaling variable $\xi=Q^{2} / 2 M \nu$, originally defined in Eq. (2):

$$
\mathcal{F}\left(\xi, \vartheta, t, Q^{2}\right)=\frac{1}{\pi} \int_{0}^{1} d \xi^{\prime}\left(\frac{1}{\xi-\xi^{\prime}-i \epsilon} \mp \frac{1}{\xi+\xi^{\prime}-i \epsilon}\right) \Im \mathrm{m} \mathcal{F}\left(\xi^{\prime}-i 0, \vartheta, t, Q^{2}\right)+\mathcal{C}_{\mathcal{F}}\left(\vartheta, t, Q^{2}\right) .
$$

For convenience, we here set the upper integration limit $\xi_{\text {cut }}=\xi_{\text {pol }}=1 /\left(1+t / 4 Q^{2}\right)$ to 1 , according to the Bjorken limit, so that the elastic pole is included in the imaginary part, rather than in the subtraction constant. We emphasize that the correct upper bound can easily be restored by the substitution $1 \rightarrow 1 /\left(1+t / 4 Q^{2}\right)$. The subtraction constant is fixed by the value of the CFFs at $\xi=\infty$. It appears in the CFFs $\mathcal{H}$ and $\mathcal{E}$, vanishes for the CFFs $\widetilde{\mathcal{H}}$ and $\widetilde{\mathcal{E}}$, and is perturbatively predicted to be zero for the combination $\mathcal{H}+\mathcal{E}$, see Ref. [34]. Thus, we can define:

$$
\mathcal{C}\left(\vartheta, t, Q^{2}\right) \equiv \mathcal{C}_{\mathcal{E}}\left(\vartheta, t, Q^{2}\right)=\mathcal{E}\left(\xi=\infty, \vartheta, t, Q^{2}\right), \quad \mathcal{C}_{\mathcal{H}}\left(\vartheta, t, Q^{2}\right)=-\mathcal{C}\left(\vartheta, t, Q^{2}\right),
$$


where $\mathcal{C}_{\widetilde{\mathcal{H}}}=\mathcal{C}_{\widetilde{\mathcal{E}}}=0$.

The dispersion relation (8) states that both the imaginary and real part of CFFs contain, up to a possible subtraction constant, the same information:

$$
\Re \mathrm{e} \mathcal{F}\left(\xi, \vartheta, t, Q^{2}\right)=\frac{1}{\pi} \mathrm{PV} \int_{0}^{1} d \xi^{\prime}\left(\frac{1}{\xi-\xi^{\prime}} \mp \frac{1}{\xi+\xi^{\prime}}\right) \Im \mathrm{m} \mathcal{F}\left(\xi^{\prime}-i 0, \vartheta, t, Q^{2}\right)+\mathcal{C}_{\mathcal{F}}\left(\vartheta, t, Q^{2}\right) .
$$

Let us loosely have a look at this statement without discussing any mathematical refinements. After extending the support of $\Im \mathrm{m} \mathcal{F}(\xi, \ldots)$ to $|\xi|>1$ and using the symmetry property, we express Eq. (10) as a (singular) Fredholm type integral equation of the first kind with constant limits $( \pm \infty)$. Briefly speaking, the real part of $\mathcal{F}(\xi, \ldots)-\mathcal{C}(\ldots)$ is the Hilbert transform of its imaginary part. The inversion reads then

$$
\Im \mathrm{m} \mathcal{F}\left(\xi, \vartheta, t, Q^{2}\right)=\frac{1}{\pi} \mathrm{PV} \int_{-\infty}^{\infty} d \xi^{\prime} \frac{1}{\xi^{\prime}-\xi}\left[\Re \mathrm{e} \mathcal{F}\left(\xi^{\prime}, \vartheta, t, Q^{2}\right)-\mathcal{C}_{\mathcal{F}}\left(\vartheta, t, Q^{2}\right)\right] .
$$

Alternatively, by again making use of the spectral condition $\Im \mathrm{m} \mathcal{F}(\xi, \ldots)=0$ for $|\xi|>1$, we can take the solution of the Fredholm type integral equation with constant limits of integration $( \pm 1)$; e.g., from Ref. [120]:

$$
\Im \mathrm{m} \mathcal{F}\left(\xi, \vartheta, t, Q^{2}\right)=\frac{\sqrt{1-\xi^{2}}}{\pi} \mathrm{PV} \int_{-1}^{1} d \xi^{\prime} \frac{1}{\sqrt{1-\xi^{2}}} \frac{\Re \mathrm{e} \mathcal{F}\left(\xi^{\prime}, \vartheta, t, Q^{2}\right)}{\xi^{\prime}-\xi} \quad \text { for } \quad|\xi| \leq 1
$$

In conclusion, assuming the specific analytic properties, we can state that the imaginary and real part of CFFs, having definite signature and a vanishing subtraction constant, have a one-toone correspondence in the 'accessible' region $0 \leq \xi \leq 1$. This correspondence seems to become imperfect in the case of non-vanishing subtraction constant (9). As in Ref. [34], we shall argue below that this might not be the case, if the $\xi \rightarrow 0$ asymptote is given by Regge behavior, as is commonly assumed.

In a real experiment one accesses only a limited phase space region. To pin down CFFs in inaccessible regions one can again employ their holomorphic property, but now expressed as a family of sum rules. To give an example, we recall that Regge asymptotics of on-shell amplitudes yield finite energy sum rules (FESRs), which are widely used in phenomenology. Pragmatically, we suppose that off-shell CFFs possess the high-energy asymptotic power-like behavior $\xi^{-\alpha(t)}$, where $\alpha(t)$ is an effective Regge trajectory, which might depend on $Q^{2}$. We introduce now a duality parameter $v$ that divides the low- $\left(v<\xi^{\prime}\right)$ and high- $\left(\xi^{\prime}<v\right)$ energy region in the dispersion 
integral (10). Expanding in the vicinity of $\xi=0$ yields FESR 12

$$
\begin{aligned}
& \int_{v}^{1} d \xi \xi^{-m-2} \Im \mathrm{m} \mathcal{F}(\xi, \cdots)=\pi S_{m+1}(v, \cdots), \text { with } \\
& S_{n}(v, \cdots)=\frac{-2^{-1}}{n !} \frac{d^{n}}{d \xi^{n}}\left[\Re \mathrm{e} \mathcal{F}(\xi, \cdots)-\mathcal{C}_{\mathcal{F}}(\cdots)-\frac{1}{\pi} \mathrm{PV} \int_{0}^{v} d \xi^{\prime}\left(\frac{1}{\xi-\xi^{\prime}} \mp \frac{1}{\xi+\xi^{\prime}}\right) \Im \mathrm{m} \mathcal{F}\left(\xi^{\prime}, \cdots\right)\right]_{\xi=0} .
\end{aligned}
$$

For odd (even) $m$ they are valid for signature $+1(-1)$. We emphasize that the $m=-1$ case deserves special attention. The resulting FESR is closely related to what is called the inverse moment. For real Compton scattering the form of this specific FESR can be found in Ref. [122].

The concealed part of the answer to the question 'What GPD information can be revealed from experiment?', arises from the analyticity of the amplitude, which ties the real and imaginary parts by the single variable dispersion relation (8), advocated in Ref. [43]. Plugging the LO result (6) for the imaginary part into the dispersion relation (8), it becomes obvious that the real part of the amplitude (4), up to a possible subtraction constant, is also expressed by the outer GPD region:

$$
\Re \mathrm{e} \mathcal{F}\left(\xi, \vartheta, t, Q^{2}\right) \stackrel{\mathrm{LO}}{=} \mathrm{PV} \int_{0}^{1} d x\left(\frac{1}{\xi-x} \mp \frac{1}{\xi+x}\right) F^{\mp}\left(x, \eta=\vartheta x, t, Q^{2}\right)+C_{F}\left(\vartheta, t, Q^{2}\right) .
$$

This 'dispersion relation' tells us that for given $|\vartheta| \leq 1$ the variation of the real part with respect to $\xi$ is governed by the value of the GPD on the trajectory $\eta=\vartheta x$. Note that we have introduced a new symbol for the subtraction constant fixed to LO:

$$
C_{F}\left(\vartheta, t, Q^{2}\right) \stackrel{\mathrm{LO}}{\equiv} \mathcal{C}_{\mathcal{F}}\left(\vartheta, t, Q^{2}\right)
$$

Compared to the LO formula (4), in which the whole GPD support contributes to the real part, the observation, manifest in Eq. (14), looks perhaps at the first sight rather surprising. It is a consequence of analyticity and the decomposition of the CFFs in terms of GPDs, which posses definite properties under Lorentz transformations, clearly visible in the operator product expansion approach (OPE) [45, 34]. The consequence is that the GPD in both the central and the outer region know about each other and talk to each other via the trajectory $\eta=\vartheta x$ [87, 1, 42, To obtain the equality that governs this cross-talk, we combine formula (14) with the LO amplitude (44) and find a family of GPD sum rules (GPDSRs) [43, 46]:

$$
\int_{0}^{1} d x\left(\frac{1}{\xi-x} \mp \frac{1}{\xi+x}\right)\left[F^{\mp}\left(x, \eta=\vartheta \xi, t, Q^{2}\right)-F^{\mp}\left(x, \eta=\vartheta x, t, Q^{2}\right)\right]=C_{F}\left(\vartheta, t, Q^{2}\right),
$$

\footnotetext{
${ }^{12}$ Assuming that $\Im \mathrm{m} \mathcal{F} \approx \pi \sum_{\alpha} \beta_{\alpha} \xi^{-\alpha} / \Gamma(\alpha+1)$ is approximately valid for $|\xi| \leq v$ one straightforwardly finds for $\alpha>0$ and $n>0: S_{n} \approx \sum_{\alpha} \frac{\beta_{\alpha}}{\Gamma(\alpha+1)} \frac{v^{-\alpha-n}}{\alpha+n}$. To obtain the textbook form of $S_{n}$ for on-shell amplitudes [52], one might now rewrite the FESR (13) in terms of the variables $\nu=Q^{2} / 2 M \xi$ and $N=Q^{2} / 2 M v$ and take instead of the Bjorken limit the on-shell one. To find the more general form, including $\alpha<0$, one should follow the lines of Ref. [121], where for $n=0$ the $\alpha=0$ case has to be treated separately, e.g., as in Ref. [122].
} 


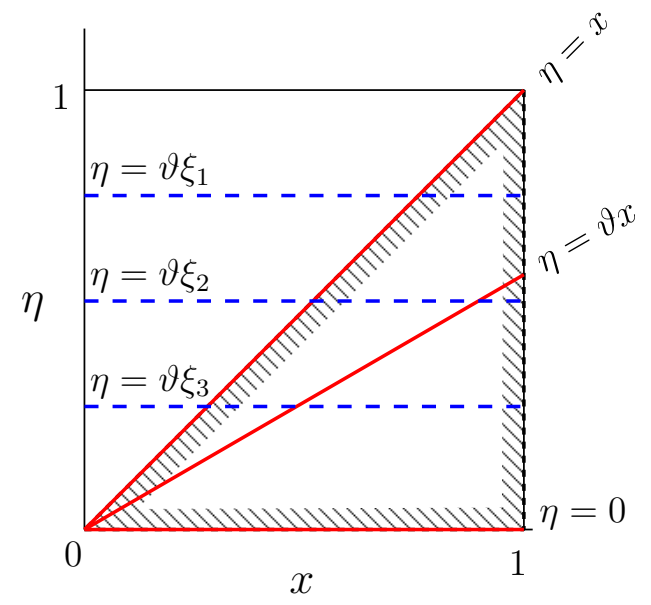

(a)

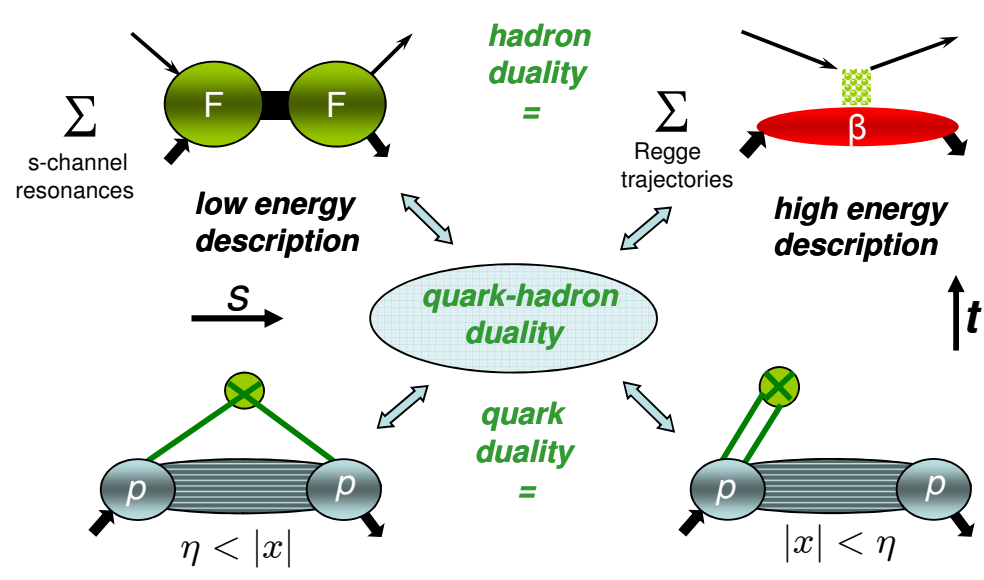

(b)

Figure 3: (a) The integration paths in the sum rule (16) along the $\eta=\vartheta x$ (solid, red) and horizontal $\eta=\vartheta \xi$ (dashed, blue) trajectories are displayed. (b) Visualization of the approximate duality relation of a GPD with the $s$ - and $t$-channel view on the hadronic amplitude. For on-shell amplitudes duality between the $s$ - and $t$-channel view has been conjectured at the end of the sixties. Quark-hadron duality was conjectured in particular in DIS, while modelling GPDs in the central region by the crossed $t$-channel contribution was proposed in [84].

parameterized by $\vartheta$. Here we have dropped the principal value prescription, since the integrand can be considered as integrable at $x=\xi$. The GPDSR family (16) is visualized in Fig. 33a. It relates the integral along the $\eta=\vartheta x$ trajectory (solid) in the outer GPD region to a family of integrals along the horizontal $\eta=\vartheta \xi$ trajectories (dashed), traversing both inner and outer regions. The variations of a spectator model GPD on a horizontal (dashed) and the cross-over trajectory (solid) are displayed in the left panel of Fig. 1.

\subsection{Deconvolution and duality}

Let us suppose that the GPD in the outer region and the subtraction constant are known from a 'measurement'. Finding the GPD in the central region can then be considered as a deconvolution problem. Decomposing the integral regions into the central and the outer one, one can view the GPDSR family (16) as a Fredholm type integral equation of the first kind, which allows one to solve the deconvolution problem. This mapping is an integral transformation that requires the 
continuation in the region $\eta>1$. Such an extension is already defined in the DD representation 13

$$
F(x, \eta, t)=(1-x)^{p} \int_{0}^{1} d y \int_{-1+y}^{1-y} d z \delta(x-y-\eta z) f(y, z, t) .
$$

One can verify that the (anti-) symmetrized GPDs within this representation satisfy the GPDSR family (16). The DD representation has been utilized in Ref. [71] to solve the deconvolution problem for a simple spectator model, formulated in the light-cone wave function overlap representation, see also Refs. [69, 70].

Within the GPDSR family (16) the deconvolution problem can also be solved by a constructive method that explicitly avoids the continuation into the region $\eta>1$. This method resembles the derivation of the standard factorization formula (4) from the short distance OPE and the dispersion relation (8) in the Euclidean region, see Refs. [45, 34].

We go now in reverse direction along the lines of Ref. [34. Expanding the integral kernel in the GPDSR family (16) in powers of $1 / \xi$ relates integral Mellin moments,

$$
\int_{-1}^{1} d x x^{j} F\left(x, \eta=\vartheta x, t, \mu^{2}\right) \quad \text { for } \quad j \geq 0
$$

of the GPD at the trajectory $\eta=\vartheta x$ to the usual Mellin moments of the GPD with argument $\eta=\vartheta \xi:$

$$
f_{j}\left(\eta, t, \mu^{2}\right)=\int_{-1}^{1} d x x^{j} F\left(x, \eta, t, \mu^{2}\right) \quad \text { for } \quad j \geq 0 .
$$

The latter are defined in terms of the expectation values of local operators with spin $j+1$. Lorentz covariance (or polynomiality condition) enforces

$$
f_{j}\left(\eta, t, \mu^{2}\right)=\sum_{\substack{n=0 \\ \text { even }}}^{j+1} f_{j}^{(n)}\left(t, \mu^{2}\right) \eta^{n}
$$

where $f_{j}^{(j+1)}=0$ for $f=\{h+e, \widetilde{h}, \widetilde{e}\}$ (for details see, e.g., [6, [7]). After reordering, we equate the coefficients in front of $\xi^{-j-1}$

$$
\int_{0}^{1} d x x^{j} F^{\mp}\left(x, \vartheta x, t, Q^{2}\right)=\sum_{\substack{n=0 \\ \text { even }}}^{\infty} \vartheta^{n} f_{j+n}^{(n)}\left(t, Q^{2}\right),
$$

and thus find the coefficients of the polynomial (19):

$$
f_{j}^{(n)}\left(t, Q^{2}\right)=\left.\frac{1}{n !} \frac{d^{n}}{d \vartheta^{n}} \int_{0}^{1} d x x^{j-n} F^{\mp}\left(x, \vartheta x, t, Q^{2}\right)\right|_{\vartheta=0} \quad \text { for } \quad j-n \geq 0,
$$

13 The factor $(1-x)^{p}$ in our representation (17) is motivated by the form of the positivity constraints [48] and appears in the model of Ref. [71]. For the GPD $E$ we have $p=1$ and thus a non-vanishing subtraction constant. For GPD $H+E$ and the two parity odd GPDs $p=0$ and the subtraction constant is absent. 
where $j=1,3,5 \cdots$ and $j=0,2,4 \cdots$ for - and + type of GPDs, respectively, and $j-n \geq 0$ with $n$ even. If a subtraction constant is present in $\mathcal{E}$, the Mellin moments with $n=j+1$ appear and are evaluated from

$$
e_{j}^{(j+1)}\left(t, Q^{2}\right)=\left.\frac{1}{2} \frac{1}{(j+1) !} \frac{d^{j+1}}{d \vartheta^{j+1}} C\left(\vartheta, t, Q^{2}\right)\right|_{\vartheta=0} .
$$

Note that $h_{j}^{(j+1)}\left(t, Q^{2}\right)=-e_{j}^{(j+1)}\left(t, Q^{2}\right)$, and that here, as in the rest of the paper, we follow the convention (9), i.e., $C \equiv C_{E}$ while $C_{H}=-C$.

All possible techniques are now at hand to construct the GPD from the moments (19), (21) and (22): standard inverse Mellin transform, expansion with respect to conformal eigenfunctions [59, 60, 61, 42, and introduction of DD as generating function for the Mellin moments [1, 72, see, e.g., Ref. [123]. All of them are based or related to the uniqueness of an analytic continuation of the even or odd Mellin moments, ensured by the Carlson theorem [124]. Thereby, the Mellin moments $e_{j}^{(j+1)}$ might either be considered connected with a 'regular part' of the GPD, e.g., as in Eq. (17), or, alternatively, they can be included in the $D$-term addenda of a GPD [94]. It is mostly a matter of taste and convenience (as long as the whole GPD is considered as known), which representation one prefers [86]. Only in the case that the spectral function, i.e., $\Im \mathrm{m} \mathcal{F}$, possesses unexpected analytic properties such as 'invisible' terms, like $x \delta(x)$, one has to treat the $e_{j}^{(j+1)}$ terms separately from the DD part. In any case, after convolution of the GPD with the hard-scattering part one has the representation for the subtraction constant, cf. Eq. (22):

$$
C\left(\vartheta, t, Q^{2}\right)=2 \sum_{\substack{j=1 \\ \text { odd }}}^{\infty} \vartheta^{j+1} e_{j}^{(j+1)}\left(t, Q^{2}\right) \quad \text { with } \quad C\left(\vartheta=0, t, Q^{2}\right)=0
$$

where the normalization at $\vartheta=0$ is the perturbative prediction. This vanishing of the subtraction constant can be considered as a consequence of the absence of a local and gauge invariant spin-zero operator in the short distance OPE.

That the GPD in the outer region can be uniquely restored from its knowledge in the central one is known and was again clearly spelled out in Ref. [42]. This mapping is (implicitly) done in the conformal partial wave expansion approach by taking appropriate boundary conditions for the partial waves; see [42, 65] for a discussion. Hence, both regions contain the same information and so they are dual to each other.

In anticipation of phenomenological applications we shall now derive GPDSRs that have the form of FESRs. Note that GPDs and their sum rules depend on the perturbative approximation and scheme conventions that are used in the factorization approach. Analogous to the the derivation of FESRs, we introduce the duality parameter $v$, which divides the integral in the GPDSR family (16) into the 'low-' and 'high-energy' contribution. By expanding the integral kernel in 
Eq. (16) around $\xi=0$ we find the desired GPDSRs:

$$
\int_{v}^{1} d x x^{-m-2}\left[F^{\mp}\left(x, \vartheta x, t, Q^{2}\right)-\left.\sum_{\substack{n=0 \\ \text { even }}}^{m+1} \frac{(\vartheta x)^{n}}{n !} \frac{d^{n}}{d \eta^{n}} F^{\mp}\left(x, \eta, t, Q^{2}\right)\right|_{\eta=0}\right]=\delta S_{m+1}^{\mp}\left(v, \vartheta, t, Q^{2}\right),
$$

where $m \geq-1$ is odd (even) for $-(+)$ type GPDs. The 'high-energy' part $\delta S_{n}^{\mp}\left(v, \vartheta, t, Q^{2}\right)$ is obtained from the Taylor coefficients:

$$
\delta S_{n}^{\mp}=\frac{-2^{-1}}{n !} \frac{d^{n}}{d \xi^{n}}\left[\int_{0}^{v} d x\left(\frac{1}{\xi-x} \mp \frac{1}{\xi+x}\right)\left[F^{\mp}(x, \vartheta \xi, \cdots)-F^{\mp}(x, \vartheta x, \cdots)\right]-C_{F}(\cdots)\right]_{\xi=0} .
$$

The 1.h.s. of the sum rule (24) relates for $x \geq v$ the GPD at the trajectory $\eta=\vartheta x$ with its behavior in the vicinity of $\eta=0$, where the strength of the skewness effect is given by its r.h.s., i.e., it is embodied in the 'high-energy' constants $\delta S_{n}^{\mp}(v, \vartheta)$.

We add that the 'low-energy' and 'high-energy' content might be related by a more elegant, however, quite formal way, using analytic (or canonical) regularization [125. This is useful if one deals with GPD models formulated in Mellin space with meromorphic functions. The GPD inherits the Regge-like behavior of the CFFs, which we assume, and should not possess any 'invisible' terms, e.g., such as $x \delta(x)$. Under this assumption one can introduce analytic regularization at $x=0$, indicated by $(0)$ as the lower integration limit, in the sum rule (16) and expand the integral kernel in the vicinity of $\xi=0$. An example is the evaluation of the subtraction constant from the 'high-energy' limit of Eq. (16):

$$
C\left(\vartheta, t, Q^{2}\right)=\lim _{\xi \rightarrow 0} \int_{0}^{1} d x \frac{2 x}{x^{2}-\xi^{2}}\left[E^{-}\left(x, \vartheta x, t, Q^{2}\right)-E^{-}\left(x, \vartheta \xi, t, Q^{2}\right)\right] .
$$

It is obvious that interchanging the limit with the integration could render a non-integrable singularity at $x=0$. Before this interchange, one first has to introduce a regularization, which does not spoil the normalization condition $C\left(\vartheta=0, t, Q^{2}\right)=0$. In the absence of 'invisible' terms, such as $x \delta(x)$, we can utilize analytic regularization and write, as in Ref. [34]:

$$
C\left(\vartheta, t, Q^{2}\right)=\int_{(0)}^{1} d x \frac{2}{x}\left[E^{-}\left(x, \vartheta x, t, Q^{2}\right)-E^{-}\left(x, 0, t, Q^{2}\right)\right] .
$$

It can easily be verified that this prescription, as well as Eq. (26), is consistent with the GPDSR (24) for $m=-1$. Under the assumption of Regge behavior, an analogous relation should hold for the imaginary part of CFFs, too, as argued in Ref. 34] based on knowledge of radiative corrections. 


\section{Phenomenological applications}

\subsection{Strategy to access GPDs}

As we saw, the imaginary part of the CFFs in the region $x=\xi \in[0,1]$ is a spectral function, considered as 'measurable' in our Compton gedanken experiment for $|\vartheta| \leq 1$. The real part, arising from the dispersion relation (8), is then immediately known, too, and it must coincide with the 'measured' one in the region $x=\xi \in[0,1]$. By means of Eq. (12), the logic can also be reversed: having restricted 'experimental access', i.e., knowing the imaginary and real part of the CFFs only in a certain limited interval, one can use the dispersion integral (8) (and also Eq. (11)) as a 'local' sum rule, which restricts the shape of the spectral function in the 'non-accessible' region. Such a restriction is possible even if one knows the real part only at one given value of $\xi$. Various forms of sum rules can be derived and we only concentrate on the FESR ones, which relate the 'low-energy' and 'high-energy' regions. 'Measuring' the spectral function in dependence of $\vartheta$, for $|\vartheta| \leq 1$, allows us to solve the deconvolution problem and to reveal the GPD. This can be expressed also in terms of GPDSRs in the form of an integral equation (16) or in terms of FESR-like ones (24).

We now apply our results to the DVCS case, in particular, to the dominant CFF $\mathcal{H}$ :

$$
\vartheta=1, \quad \mathcal{H}\left(\xi, t, \mathcal{Q}^{2}\right) \equiv \mathcal{H}\left(\xi, \vartheta=1, t, Q^{2}\right), \quad \mathcal{C}\left(\xi, t, \mathcal{Q}^{2}\right) \equiv \mathcal{C}\left(\xi, \vartheta=1, t, Q^{2}\right)
$$

Here we introduce the virtuality of the photon $\mathcal{Q}^{2} \approx 2 Q^{2}$. Everything that was stated regarding the measurement of CFFs remains valid in DVCS kinematics. It is often easier to measure the imaginary part (via single spin asymmetries or polarized cross sections) than the real part of the amplitude. Hence, one might use the available experimental findings for the real part to reveal the imaginary part of the CFF outside of the accessible phase space.

We introduce two phenomenological parameters $\underline{\eta}$ and $\bar{\eta}$ to divide the $\xi$ interval into the small $(\xi \leq \underline{\eta})$, valence $(\underline{\eta} \leq \xi \leq \bar{\eta})$, and large $(\bar{\eta} \leq \xi)$ region. We assume that the CFF can be measured in the valence region $\underline{\eta} \leq \xi \leq \bar{\eta}$. The dispersion integral (8) might then be used to constrain the imaginary part in the large $\xi^{\prime}$ region $\bar{\eta} \leq \xi^{\prime}$

$$
\int_{\bar{\eta}}^{1} d \xi^{\prime} \frac{2 \xi^{\prime}}{\xi^{2}-\xi^{\prime 2}}\left[\Im \mathrm{m} \mathcal{H}\left(\xi^{\prime}, t, \mathcal{Q}^{2}\right)-\Im \mathrm{m} \mathcal{H}\left(\xi, t, \mathcal{Q}^{2}\right)\right]=\pi \mathcal{S}^{\exp }\left(\xi, t, \mathcal{Q}^{2} \mid \bar{\eta}, \underline{\eta}\right)+\pi \mathcal{S}^{\bmod }\left(\xi, t, \mathcal{Q}^{2} \mid \underline{\eta}\right),
$$

from the experimental knowledge of the region $\underline{\eta} \leq \xi^{\prime} \leq \bar{\eta}$ :

$$
\begin{aligned}
\mathcal{S}^{\exp }(\xi, t, \mathcal{Q} \mid \bar{\eta}, \underline{\eta})= & \Re \mathrm{e} \mathcal{H}\left(\xi, t, \mathcal{Q}^{2}\right)-\frac{1}{\pi} \ln \left(\frac{\xi^{2}}{1-\xi^{2}}\right) \Im \mathrm{m} \mathcal{H}\left(\xi, t, \mathcal{Q}^{2}\right) \\
& +\frac{1}{\pi} \int_{\underline{\eta}}^{\bar{\eta}} d \xi^{\prime} \frac{2 \xi^{\prime}}{\xi^{\prime 2}-\xi^{2}}\left[\Im \mathrm{m} \mathcal{H}\left(\xi^{\prime}, t, \mathcal{Q}^{2}\right)-\Im \mathrm{m} \mathcal{H}\left(\xi, t, \mathcal{Q}^{2}\right)\right]
\end{aligned}
$$


the model estimate for the subtraction constant, and the extrapolation into the small $\xi^{\prime}$ region $\xi^{\prime} \leq \underline{\eta}:$

$$
\mathcal{S}^{\bmod }(\xi, t, \mathcal{Q} \mid \underline{\eta})=\mathcal{C}\left(t, \mathcal{Q}^{2}\right)+\frac{1}{\pi} \int_{0}^{\underline{\eta}} d \xi^{\prime} \frac{2 \xi^{\prime}}{\xi^{\prime 2}-\xi^{2}}\left[\Im \mathrm{m} \mathcal{H}\left(\xi^{\prime}, t, \mathcal{Q}^{2}\right)-\Im \mathrm{m} \mathcal{H}\left(\xi, t, \mathcal{Q}^{2}\right)\right] .
$$

The subtraction constant is known to some extent, see, e.g., Refs. [74, 100, 83], and one might develop some understanding how Regge phenomenology should be employed for off-shell processes. In evaluating the amount arising from the very small $\xi$ region one can use the DVCS measurements of the H1/ZEUS collaborations [9, 12, 13, 18]. Hence, the model dependent part is therefore somewhat under control. The large $\xi$ region which one reveals in this way is certainly challenging to measure in experiment. As one realizes, even knowledge of the $\mathrm{CFF}$ at one value of $\xi$, i.e., $\underline{\eta}=\bar{\eta}=\xi$, leads to a highly non-trivial constraint. This sum rule (29) (with $\xi \leq \bar{\eta}$ ) can again be considered as a family of FESR. To see this, one simply expands the integral kernel with respect to $\left(\xi / \xi^{\prime}\right)^{2}$. We also note that the real part of the CFF $\mathcal{H}$ can be measured in the crossed channel $\gamma^{*} \gamma^{(*)} \rightarrow N \bar{N}$ (or $N \bar{N} \rightarrow l^{+} l^{-} \gamma$ ) and then obtained by analytic continuation.

The dispersion integral and crossing symmetry allows for revealing the CFF as a physical quantity. The microscopic view on this quantity is given by perturbation theory, providing us, to LO accuracy, with a partonic interpretation in terms of the GPD on the cross-over trajectory:

$$
H^{-}\left(x, x, t, \mathcal{Q}^{2}\right) \stackrel{\text { LO }}{=} \frac{1}{\pi} \Im \mathrm{m} \mathcal{H}\left(\xi=x, t, \mathcal{Q}^{2}\right) \quad \text { for } \quad \underline{\eta} \leq x \leq \bar{\eta} .
$$

To that accuracy we write the physical sum rule (29) as a GPD one:

$$
\begin{aligned}
& \int_{\bar{\eta}}^{1} d x \frac{2 x}{\xi^{2}-x^{2}}\left[H^{-}\left(x, x, t, \mathcal{Q}^{2}\right)-H^{-}\left(\xi, \xi, t, \mathcal{Q}^{2}\right)\right]-C\left(t, \mathcal{Q}^{2}\right) \\
+ & \int_{0}^{\underline{\eta}} d x \frac{2 x}{\xi^{2}-x^{2}}\left[H^{-}\left(x, x, t, \mathcal{Q}^{2}\right)-H^{-}\left(\xi, \xi, t, \mathcal{Q}^{2}\right)\right] \stackrel{\text { LO }}{=} \mathcal{S}^{\exp }(\xi, t, \mathcal{Q} \mid \bar{\eta}, \underline{\eta}) \quad \text { for } \quad \underline{\eta} \leq \xi \leq \bar{\eta} .
\end{aligned}
$$

It clearly states that in an experiment the GPD can be accessed only on the cross-over trajectory.

The information which can be revealed away from the cross-over trajectory is governed by the GPDSR family (16), which we write in the form of an integral equation

$$
\mathrm{PV} \int_{0}^{1} d x \frac{2 x}{\eta^{2}-x^{2}} H^{-}\left(x, \eta, t, \mathcal{Q}^{2}\right)=\mathrm{PV} \int_{0}^{1} d x \frac{2 x}{\eta^{2}-x^{2}} H^{-}\left(x, x, t, \mathcal{Q}^{2}\right)-C\left(t, \mathcal{Q}^{2}\right) .
$$

The r.h.s. of this integral equation is considered as known and can be equated in an ideal experiment with the measured real part of the CFF (including the subtraction constant). The l.h.s. is nothing more than the real part of the LO result (44). In addition, we have the 'boundary condition' that $H^{-}\left(x, x, t, Q^{2}\right)$ is equal to the imaginary part of the CFF. Knowing the imaginary and real part of $\mathcal{H}$ in an ideal experiment, one would be able to measure the subtraction constant. However, the deconvolution problem cannot be solved 14 within the family of GPDSRs (34).

\footnotetext{
${ }^{14}$ We recall that the scale dependence provides an additional handle on the deconvolution problem.
} 
The invisible terms in an ideal DVCS experiment are the zero modes,

$$
\int_{0}^{1} d x \frac{2 x}{\eta^{2}-x^{2}} \delta H\left(x, \eta, t, \mathcal{Q}^{2}\right) \equiv 0
$$

in the integral equation (34) with the boundary conditions

$$
\delta H\left(x, \eta=x, t, \mathcal{Q}^{2}\right)=0, \quad \lim _{\eta \rightarrow 0} \delta H\left(x, \eta, t, \mathcal{Q}^{2}\right)=x^{\epsilon}, \quad \lim _{\eta \rightarrow 1} \delta H\left(x, \eta, t, \mathcal{Q}^{2}\right)=(1-x)^{\bar{\epsilon}},
$$

where $\{\underline{\epsilon}, \bar{\epsilon}\}>0$. Due to the duality properties, governed by Lorentz covariance, it is clear that a priori any given function in the outer or central region which satisfies the boundary conditions defines a zero mode. A simple example for fixed $\mathcal{Q}^{2}$ is

$$
\delta H^{\text {toy }}(x, \eta, t)=\frac{\left(\eta^{2}-x^{2}\right)}{|x|} \frac{d}{d x} f(|x|, t)
$$

with appropriate behavior of $f(x, t)$ at the boundaries $x=0$ and $x=1$. Hence, the experimental data (at least for a fixed scale) can be described by numerous GPD models. In our toy example, the zero mode might contribute to the forward case and might be visible for $t=0$ in DIS. An example of a term visible in DVCS, but invisible in forward kinematics, is given in Ref. [25]. Also, for GPD $E^{-}$, where phenomenological constraints for the forward limit do not exist, any GPD model which fits the data is as good as any other, obtained by a 'gauge' transformation. However, the zero mode might contribute to the first moment:

$$
E^{-\mathrm{fit}}\left(x, \eta, t, \mathcal{Q}^{2}\right) \rightarrow E^{-\mathrm{fit}}\left(x, \eta, t, \mathcal{Q}^{2}\right)+\delta E\left(x, \eta, t, \mathcal{Q}^{2}\right), \quad \int_{-1}^{1} d x x \delta E\left(x, \eta, t, \mathcal{Q}^{2}\right) \neq 0 .
$$

Obviously, different GPD models which are able to describe the experimental data can provide different values for the desired first moment, which enters the quark angular momentum sum rule [8].

Although the GPDSR family (34) states that the central and the outer GPD region are related via the GPD on the cross-over trajectory, some information might be lost in DVCS and/or for forward kinematics. For a more geometrical interpretation we refer to the discussion in the Introduction and Fig. 1(b). Only in the case that for GPDs the holographic principle applies, i.e., all information which is contained in the GPD can be obtained from its value on the cross-over trajectory, the whole GPD can be revealed from an (ideal) measurement. Nevertheless, the GPD sum rules (34) provide us with valuable information on the $\eta$-dependence of the GPD.

This can be seen if we decompose the integral into contributions from the central and outer regions. Neglecting a possible subtraction constant and assuming that the Regge behavior is $\eta$-independent,

$$
\int_{0}^{\eta} d x \frac{2 x}{\eta^{2}-x^{2}}\left[H^{-}(x, x, t)-H^{-}(x, \eta, t)\right]=\int_{\eta}^{1} d x \frac{2 x}{x^{2}-\eta^{2}}\left[H^{-}(x, x, t)-H^{-}(x, \eta, t)\right],
$$


we find that in the limit $\eta \rightarrow 0$ the l.h.s. vanishes and the r.h.s. must therefore satisfy an inverse moment sum rule. Taking now the limit $\eta \rightarrow 1$ and using the fact that the GPD is vanishing at the end point $x=1$, we find another sum rule. These sum rules read

$$
\int_{0}^{1} d x \frac{1}{x}\left[H^{-}(x, x, t)-H^{-}(x, 0, t)\right]=0, \quad \int_{0}^{1} d x \frac{x}{1-x^{2}}\left[H^{-}(x, x, t)-H^{-}(x, 1, t)\right]=0 .
$$

In the left sum rule (i.e., $\eta \rightarrow 0$ ) the GPD on the cross-over trajectory is dictated by the physical content in the outer region, while in the right sum rule (i.e., $\eta \rightarrow 1$ ) it is governed by the one in the central region. This suggests that for small $\eta$ we shall naturally have the partonic $s$-channel point of view, while for large $\eta$ the $t$-channel point of view in terms of meson-like exchanges is more appropriate [84]; see left and right sketch in the lower part of the Fig. 3(b), respectively. To match both regions one might sum up the $t$-channel exchanges, i.e., reggeize them. If one would like to incorporate phenomenological knowledge of hadronic physics in GPD modelling, one might suppose that for small $x$ and $\eta$ one understands better the hadronic $t$-channel view in terms of mesonic Regge exchanges [34, while for large $\eta$ and $x$ the $s$-channel partial wave expansion in terms of few baryonic resonances is more useful [127]; see right and left sketch in the upper part of the Fig. 3(b), respectively. Hence, GPD modelling from phenomenological input should be related as indicated across the four sketches in the Fig. 3(b) rather then vertically. Finally, we note that the left equation in Eq. (40) is given in its more general form in Eq. (49).

Let us also discuss the problem of relating the GPD at the cross-over trajectory with the zero skewness GPD from the point of view of Mellin moments. The subtraction constant that enters the DVCS process is, according to Eq. (23), given by

$$
C\left(t, \mathcal{Q}^{2}\right)=-2 \sum_{\substack{j=1 \\ \text { odd }}}^{\infty} h_{j}^{(j+1)}\left(t, \mathcal{Q}^{2}\right)
$$

A model dependent estimate of the constant for $t=0$ was provided in $\chi$ QSM [74]. The $\chi$ QSM model evaluation of the first term in the series (41), given in Ref. [100], is compatible with lattice measurements 83 . This allows one to estimate the size of the constant $C\left(t, \mathcal{Q}^{2}\right)$, to judge on the convergence of the series (41), and to estimate its $t$-dependence. According to Eq. (20), the Mellin moments of the GPD at the cross-over trajectory are given by the series

$$
\int_{0}^{1} d x x^{j} H^{-}\left(x, x, t, \mathcal{Q}^{2}\right)=\sum_{\substack{n=0 \\ \text { even }}}^{\infty} h_{j+n}^{(n)}\left(t, \mathcal{Q}^{2}\right) .
$$

The first term with $n=0$ in this series is the $j^{\text {th }}$ Mellin moment of the GPD at $\eta=0$.

Instead of using the number of total derivatives $n$ as a label for the degrees of freedom, one might prefer the angular momentum $J$ of the $t$-channel $\mathrm{SO}(3)$ partial wave expansion, which is 
a physical degree of freedom. If we apply crossing symmetry to the GPDSR family (34), for details see, e.g., Ref. [42], and set $\eta^{2} \approx 1 / \cos ^{2} \theta$, where $\theta$ is the scattering angle in the $t$-channel center-of-mass frame, we obtain the LO approximation of the corresponding $\gamma \gamma^{*} \rightarrow p \bar{p}$ amplitude in terms of the generalized distribution amplitudes $H^{(t)-}\left(z, \cos \theta, s, \mathcal{Q}^{2}\right)$ [1, 85]:

$$
\int_{0}^{1} d z \frac{2 z}{1-z^{2}} H^{(t)-}\left(z, \cos \theta, s, \mathcal{Q}^{2}\right)=\int_{0}^{1} d x \frac{2 x \cos ^{2} \theta}{1-x^{2} \cos ^{2} \theta} H^{-}\left(x, x, t, \mathcal{Q}^{2}\right)-\left.C\left(t, \mathcal{Q}^{2}\right)\right|_{t \rightarrow s} .
$$

In the $\mathrm{CFF}$ combination $\mathcal{H}+t \mathcal{E} / 4 M_{N}^{2}$ Legendre polynomials $P_{J}(\cos \theta)$ with angular momentum $J=0,2,4, \cdots$ are the proper partial waves [6]. The corresponding partial wave amplitudes $a_{J}\left(s, \mathcal{Q}^{2}\right)$ can be obtained from the orthogonality relation for Legendre polynomials. In other words, one multiplies the l.h.s. or r.h.s. of, e.g., Eq. (43) with the partial waves and integrates over $Z=\cos \theta$. It is then clear from Eq. (43) that the subtraction constant $C\left(s, \mathcal{Q}^{2}\right)$ contributes only to the $J=0$ partial wave amplitude. For $J \geq 2$ the r.h.s. of (43) tells us that the partial wave amplitudes can be obtained from the Froissart-Gribov projection by analytic continuation in $t$ :

$$
\left.a_{J}\left(s, \mathcal{Q}^{2}\right) \stackrel{\mathrm{LO}}{=} 2 \int_{0}^{1} d x \frac{\mathcal{Q}_{J}(1 / x)}{x^{2}}\left[H^{-}\left(x, x, t, \mathcal{Q}^{2}\right)+\frac{t}{4 M_{N}^{2}} E^{-}\left(x, x, t, \mathcal{Q}^{2}\right)\right]\right|_{t \rightarrow s},
$$

where $\mathcal{Q}_{J}(z)$ are the Legendre functions of the second kind. The representation (44) is suited for the analytic continuation in $J$ within $\alpha(t)<\Re \mathrm{e} J$, where $\alpha(t)$ is the leading Regge trajectory. The partial wave amplitudes are now, via Eq. (44), represented in terms of partonic quantities, namely, by the GPD on its cross-over trajectory. We might also express them as a series of common Mellin moments, labelled by the spin and the number of total derivatives, or by the moments with definite conformal spin [34].

Obviously, the perturbative approach 'predicts' the partial wave amplitudes in terms of GPDs. Since the GPDs are unknown, it is more appropriate to employ the reverse logic. As far as the partial wave amplitudes are known they provide a strong constraint on the GPDs. A power-like parameterization of the GPDs at its cross-over trajectory at small $x$, i.e., $x^{-\alpha(t)}$, corresponds to a pole at $J=\alpha(t)$ in the $\mathrm{SO}(3)$ partial wave amplitudes and makes direct contact to Regge phenomenology, extended to off-shell kinematics. For integral values of $J \geq 2$ we can read Eq. (44) as a sum rule. The case $J=0$ deserves special attention. It can be unproblematically evaluated from Eq. (43). Naively setting $J=0$ in Eq. (44) provides a divergent integral as the natural outcome of our standard derivation. We should require that both $J=0$ results are equal, which finally would provide a definition of the inverse moment for the GPD at its cross-over trajectory. Such an inverse moment is then simply given by the subtraction constant $C\left(t, \mathcal{Q}^{2}\right)$.

The GPDSRs, such as in Eq. (34), might be applied in various manners and we have presented here only a few illustrative examples. Finally, we would like to point out that they also make contact to the work of Refs. [84, 40, 41]. Reducing the GPDSRs (24) to the DVCS case, i.e., 
$\vartheta=1$, we find in the limit $v \rightarrow 0$ :

$$
\int_{0}^{1} d x\left[x^{-m-2} F^{\mp}\left(x, x, t, Q^{2}\right)-\left.\sum_{\substack{n=0 \\ \text { even }}}^{m+1} \frac{x^{n-m-2}}{n !} \frac{d^{n}}{d \eta^{n}} F^{\mp}\left(x, \eta, t, Q^{2}\right)\right|_{\eta=0}\right]=\lim _{v \rightarrow 0} \delta S_{m+1}^{\mp}\left(v, t, \mathcal{Q}^{2}\right),
$$

where $S_{m+1}^{\mp}\left(v, t, \mathcal{Q}^{2}\right)=S_{m+1}^{\mp}\left(v, \vartheta=1, t, Q^{2}\right)$ can be read off from Eq. (25). If we now view all terms as analytic functions of $m$, we can invert this specific GPDSR (45), by an inverse Mellin transform to express the GPD at its cross-over trajectory in terms of 'low-', i.e., second term in the square brackets of Eq. (45), and 'high-energy' constants, i.e., the terms of $S_{m+1}^{\mp}$. This can then also be converted in a series of forward-like parton distributions $q_{\nu}\left(x, t, \mathcal{Q}^{2}\right)$ weighted with $x^{\nu}$. The expansion of the GPD on its cross-over trajectory in this forward-like parton distributions can be written, e.g., for $H^{-}$, a: 15

$$
H^{-}\left(x, x, t, \mathcal{Q}^{2}\right)=\sum_{\substack{\nu=0 \\ \text { even }}}^{\infty} x^{\nu} q_{\nu}\left(x, t, \mathcal{Q}^{2}\right) \quad \text { with } \quad q_{0}\left(x, t, \mathcal{Q}^{2}\right) \equiv H^{-}\left(x, \eta=0, t, \mathcal{Q}^{2}\right) .
$$

Here the forward-like parton functions know about both the 'low-' and 'high-energy' part and should be viewed as generalized functions in the mathematical sense. The polynomiality condition translates into

$$
\int_{0}^{1} d x x^{m-1} q_{n}\left(x, t, \mathcal{Q}^{2}\right)=0 \quad \text { for } \quad 2 \leq m<n,
$$

where $m$ and $n$ are even. The decomposition of these forward-like parton functions in forward-like conformal or 'dual' parton functions has been discussed in the small $\eta$ expansion in Ref. [126]. Generally, it should be given by an integral transformation, cf. Refs. [60, 61, 84, 40, 41]. We emphasize that these forward-like functions, e.g., given by explicit expressions in Ref. [41], can be interpreted in terms of GPDSRs (45). The DVCS amplitude to LO in the 'dual' parameterization is then finally represented as a sum of functions that are associated with the 'low-' and 'highenergy' content, where the duality scale $v$ itself is taken to be zero; see Ref. [41]. This step generates the $\delta$-functions (including derivatives) which appear in the 'dual' parameterization. We recall that in the series (46) all forward-like parton distributions contribute to the small $x$ region and that at least the first two terms are needed to describe DVCS to LO accuracy in the small $x_{\mathrm{Bj}}$ region.

\footnotetext{
${ }^{15}$ One might find this result directly from the 'Taylor expansion' of $H^{-}\left(x, \eta, t, \mathcal{Q}^{2}\right)$ in the vicinity of $\eta=0$ with $x=\eta$. However, the expansion coefficients are generalized functions, whose property (47) ensures polynomiality. These forward-like parton distributions can be straightforwardly evaluated from DDs and the expansion (46) can be studied, e.g., within a RDDA.
} 


\subsection{Revealing GPDs from experimental measurements}

We address now the question: "What do we learn from a DVCS measurement?". To have a partonic interpretation one is mostly interested in the GPD at $\eta=0$. The extrapolation of $F^{\mp}\left(x, x, t, \mathcal{Q}^{2}\right)$ to $F^{\mp}\left(x, 0, t, \mathcal{Q}^{2}\right)$ is a model dependent procedure, which can be parameterized by the skewness function $S\left(x, t, \mathcal{Q}^{2} \mid F^{\mp}\right)$ defined by

$$
F^{\mp}\left(x, x, t, \mathcal{Q}^{2}\right)=\left[1+S\left(x, t, \mathcal{Q}^{2} \mid F^{\mp}\right)\right] F^{\mp}\left(x, \eta=0, t, \mathcal{Q}^{2}\right) .
$$

Note that we included a $t$-dependence in the skewness function, since it is expected that this dependence at large $x$ is rather different from that of the GPD at $\eta=0$. The GPDSR family (16) provides us in the limit $\xi \rightarrow 0$ with a link between the measured GPD at $\eta=x$ and $\eta=0$ and therefore a constraint for the skewness function. Since the limit might be intricate, we first have to regularize:

$$
\int_{(0)}^{1} d x \frac{1}{x} S\left(x, t, \mathcal{Q}^{2} \mid F^{-}\right) F^{-}\left(x, \eta=0, t, \mathcal{Q}^{2}\right)=\frac{1}{2} C_{F}\left(t, \mathcal{Q}^{2}\right) .
$$

One might consider the $F^{\mp}\left(x, x, t, \mathcal{Q}^{2}\right)$ as new functions, which are in an unknown manner connected to the GPD at $\eta=0$. As emphasized above, the analytic regularization procedure might be reinterpreted in terms of partonic FESR. The constraint (49) on the skewness effect is rather weak; only the inverse moment is constrained, and so there are infinite degrees of freedom left, e.g., in the series (41). We might parameterize the skewness effect by relative deviation factors:

$$
\delta_{j}\left(t, \mu^{2} \mid F^{\mp}\right) \equiv \sum_{\substack{n=2 \\ \text { even }}}^{\infty} \frac{f_{j+n}^{(n)}\left(t, \mu^{2}\right)}{f_{j}\left(t, \mu^{2}\right)}=\frac{\int_{0}^{1} d x x^{j} S\left(x, t, \mu^{2} \mid F^{\mp}\right) F^{\mp}\left(x, \eta=0, t, \mu^{2}\right)}{\int_{0}^{1} d x x^{j} F^{\mp}\left(x, \eta=0, t, \mu^{2}\right)}
$$

with, as before, $j$ being odd (even) for the $-(+)$ superscripts. The Mellin moments of CFFs are then given as a product,

$$
\int_{0}^{1} d \xi \xi^{j} \Im \mathrm{m} \mathcal{F}\left(\xi, t, \mathcal{Q}^{2}\right) \stackrel{\mathrm{LO}}{=} \pi \int_{0}^{1} d \xi \xi^{j} F^{\mp}\left(x=\xi, \xi, t, \mathcal{Q}^{2}\right)=\pi f_{j}\left(t, \mathcal{Q}^{2}\right)\left[1+\delta_{j}\left(t, \mathcal{Q}^{2} \mid F^{\mp}\right)\right]
$$

of the desired Mellin moment $f_{j}\left(t, \mathcal{Q}^{2}\right)$, surviving the forward limit, times the skewness effect.

For $t=0$ the Mellin moments $h_{j}\left(t=0, \mathcal{Q}^{2}\right)\left[\widetilde{h}_{j}\left(t=0, \mathcal{Q}^{2}\right)\right]$ are those of the PDFs and are constrained by phenomenological input. The lowest Mellin moments $f_{j=0}\left(t, \mathcal{Q}^{2}\right)$ are partonic form factors, related to observables. The deviation factors are built by a series (50) and the present state of the art in lattice measurements, to the best of our knowledge, allows for the evaluation of the first term in this series for $j=0$, i.e., the quantity $h_{2}^{(2)}$. As long as the forward limit is known (to some extent) a DVCS measurement of, e.g., $\mathcal{H}$ allows us to pin down the skewness effect for 
this quantity. In the case one wants to extract, for instance, the quark angular momentum in an ideal DVCS experiment via the anomalous gravitomagnetic moment [8],

$$
B\left(\mathcal{Q}^{2}\right) \equiv e_{1}\left(t=0, \mathcal{Q}^{2}\right) \stackrel{\mathrm{LO}}{=} \frac{1}{\pi} \frac{\int_{0}^{1} d \xi \xi \lim _{t \rightarrow 0} \Im \mathrm{m} \mathcal{E}\left(\xi, t, \mathcal{Q}^{2}\right)}{1+\delta_{1}\left(t=0, \mathcal{Q}^{2} \mid E^{-}\right)}
$$

one certainly needs to know the skewness effect (at $t=0$ ), which might be different from that appearing in $\mathcal{H}$.

The conclusion is obvious. One must use additional information to reveal the quantities one likes to pin down. Lattice measurements can give only a hint on the size of the deviation factors, and this we consider very valuable. Model evaluations, e.g., within $\chi$ QSM, can at least give estimates for the size of deviation factors. As in any of such model calculation, the matching of collective with current quark degrees of freedom induces uncertainties. Nevertheless, it is very important to quantify these deviation factors on a generic level. Further experimental constraints on them, available in the small $x_{\mathrm{Bj}}$ region, arise from the logarithmical scaling violation, governed by evolution. A discussion of revealing the quark angular momentum from the present DVCS data should be given elsewhere. We would only like to note here that GPD model dependent constraints on the quark angular momentum require understanding of the skewness effect. A minimal basic requirement for such a constraint is that the utilized GPD model describes all available DVCS data, including the presently challenging data on unpolarized cross section.

According to the above discussion, one strategy for extracting GPD information to LO accuracy might consist of the following steps: (suppose that we consider $H$ or $\widetilde{H}$ )

- model the $t$-dependency of the GPD at $\eta=0$,

- parameterize the skewness function, defined in Eq. (48), and

- fit the parameters to the measured observables (CFFs) by means of Eqs. (32) and (33).

Alternatively, in the third step one might use the sum rule (29) to first pin down the imaginary part in the whole region from knowledge of the real part and then reveal from Eq. (32) the GPD at the cross-over trajectory.

Certainly, in the first step it is crucial to have a realistic model for the interplay of the $x$ - and $t$-dependence of the $\eta=0$ GPD. Proposals for the functional form of the GPD at $\eta=0$ are given in Ref. [128, 129, 91. We would like to suggest another empirical recipe that allows one to take advantage of theoretical/phenomenological knowledge on the generic level. Moreover, it has a power-like behavior at large $-t$ and can in principle also be used for positive values of $t$. Inspired by the simplicity of the Veneziano model [130] for on-shell amplitudes, we found it simple to set 

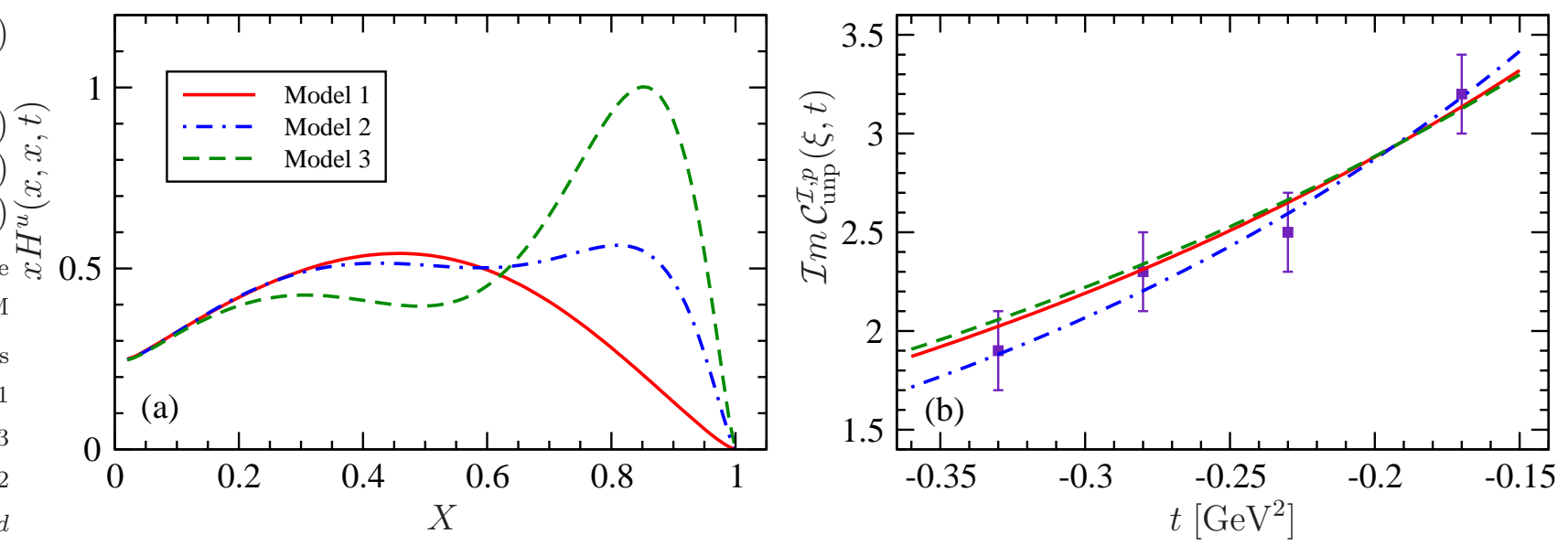

Figure 4: Panel (a) shows various models for the dominant $u$-quark GPD $H$ at $\eta=x$ versus the Bjorken-like variable $X=2 x /(1+x)$ for fixed $t=-0.25 \mathrm{GeV}^{2}$. In panel (b) we confront these models with JLab/Hall A measurements of the observable $\Im m \mathcal{C}_{\text {unp }}^{\mathcal{I}, p}$, defined in Eq. (54), for DVCS off unpolarized proton [14] for $x_{\mathrm{Bj}}=0.36$ and $\mathcal{Q}^{2}=1.9 \mathrm{GeV}^{2}$.

up GPD models at $\eta=0$ in Mellin space by taking ratios of $\Gamma$ functions, e.g., for the valence $d$-quark contribution:

$$
h_{j}^{d_{\text {val }}}=\frac{\Gamma(1+j-\alpha(t)) \Gamma(1+j+P-\alpha) \Gamma(2-\alpha+\beta)}{\Gamma(1-\alpha) \Gamma(1+j+P-\alpha(t)) \Gamma(2+j-\alpha+\beta)} .
$$

The generic values of the parameters arise from counting rules $(\beta=3, P=2)$, Regge phenomenology $\left(\alpha(t)=1 / 2+t / \mathrm{GeV}^{2}\right)$, and for $u$-quarks we employ in addition $\mathrm{SU}(6)$ symmetry arguments, i.e., $h_{j}^{u_{\text {val }}} / h_{j}^{d_{\text {val }}}=5$ for $j \rightarrow \infty$. These parameters are then understood as effective ones and are slightly tuned to phenomenological PDF parameterizations and form factor measurements. Lattice results [83] can be quantitatively reproduced within our ansatz if we adopt the found parameterization of the Regge trajectory $\alpha(t)$ to the large meson mass lattice world 16 The guidance for the parameterization of the skewness function $S\left(x, t, \mathcal{Q}^{2}=2 \mathrm{GeV}^{2} \mid H^{-}\right)$comes from the functional form of the solution for the positivity constraints [50], the expected large $x$ behavior for GPDs at $\eta=x$ [131], and model results [71]. The skewness function satisfies the sum rule (49), where the $t$-dependence of the subtraction constant has been taken from the first coefficient of the $D$-term from the $\chi$ QSM result [100]. The small $x$ behavior of the GPD $H$ is taken from our LO fits to the H1/ZEUS data [88]. The modelling will be described in detail somewhere else.

In Fig. 4(a) we show various versions of the model for the dominant $u$-quark GPD $H$. Only the solid curve is rather similar to the model versions of Ref. [27; the two other ones belong to a

\footnotetext{
${ }^{16}$ We do not share the wide-spread belief, e.g., in Ref. [89], that present lattice data should be used as quantitative GPD constraints.
} 
new class of GPD models. As argued in Sect. 2.2.3, they are enhanced in the large $X=2 x /(1+x)$ region. Note that such a functional form resembles the shape of the energy spectrum function of an exclusive process, if $1 / \mathrm{X}$ is viewed as a rescaled energy (perhaps one would even expect a resonance structure before the 'continuum' starts). The $u$-quark GPD $H$ is the dominant one in DVCS off an unpolarized proton target and it might be revealed from the azimuthal dependence of the interference term. The quantity which determines the magnitude of the dominant first harmonic in the interference term [27],

$$
\mathcal{C}_{\text {unp }}^{\mathcal{I}}=F_{1}(t) \mathcal{H}\left(\xi, t, \mathcal{Q}^{2}\right)+\xi\left[F_{1}(t)+F_{2}(t)\right] \widetilde{\mathcal{H}}\left(\xi, t, \mathcal{Q}^{2}\right)-\left.\frac{t}{4 M^{2}} F_{2}(t) \mathcal{E}\left(\xi, t, \mathcal{Q}^{2}\right)\right|_{\xi=\frac{x_{\mathrm{Bj}}}{2-x_{\mathrm{Bj}}}},
$$

is shown in Fig. 4(b) for JLab/Hall A kinematics:

$$
x_{\mathrm{Bj}}=0.36, \mathcal{Q}^{2}=1.9 \mathrm{GeV}^{2} \text {, and } 0.15 \mathrm{GeV}^{2} \leq-t \leq 0.35 \mathrm{GeV}^{2} .
$$

The relative contribution of the terms involving CFFs $\widetilde{\mathcal{H}}$ and $\mathcal{E}$ we estimate to be on the level of $10 \%-40 \%$ and up to $\pm 10 \%$, respectively, compared to the dominant $\mathcal{H}$ term. Instead of exploiting the sum rule family (29), we confronted our GPD model with experimental data in the old fashioned way. We varied the unknown contributions and compensated for this by tuning the skewness function for $H$. We emphasize that any curve in Fig. 4(a) that crosses one of the shown ones at $X=0.36$ defines a priori a GPD model, which is not ruled out by the JLab/Hall A data in panel (b). The GPD models are then selected by confronting them with the measurement for the real part for the observable (54). We found that the extracted values for this quantity are contaminated by DVCS squared terms and so there is a further contribution from the GPD $\widetilde{E}$. Taking all this into account we had no problems to tune the model parameters to the measured values for $t=-0.25 \mathrm{GeV}^{2}$. However, we were only able to get a qualitative agreement with the measured $t$-dependence of this quantity. Whether this is an artifact of our own GPD model restrictions or is due to the power suppressed terms in the twist-three formalism of Ref. [27] remains for the moment an open question. This is in our opinion more a technicality than an issue, which, of course, must be investigated.

\section{Outlook}

We have considered CFFs as holomorphic functions and discussed the phenomenological application of this property. To LO accuracy and for fixed photon virtuality one can in a DVCS measurement access the GPD only on its cross-over trajectory $\eta=x$. Combining dispersion relation and perturbation theory allows for a derivation of the partonic analogue of FESR, which relate the small and large $x$ behavior of the GPD. Altogether, this sets up a new strategy for revealing GPDs from experimental DVCS measurements, utilizing well-known techniques from the 
phenomenology of on-shell hadronic processes. The proposed alternative LO GPD approach has numerous advantages with respect to the present ad hoc GPD model approach. The LO GPDs can be considered as 'super' GPDs that contain all perturbative and non-perturbative corrections. If the assumed holomorphic properties hold true, the approach by definition cannot fail to describe DVCS data for fixed photon virtuality. All discussions about GPD models/ansätze and erroneous conclusions coming from their success or failure are avoided from the very beginning. The goal of this concept is to reveal GPDs at their cross-over trajectory $\eta=x$ only from DVCS data and to obtain a generic understanding of the skewness effect.

The partonic interpretation of the GPD to LO accuracy has been given here only from the principal point of view, where the appropriate choice of scaling variables was not discussed. This rather important problem is related to the minimization of not well-known higher twist contributions, by appropriate variable definitions. We recall that a partonic interpretation of the 'measured' GPD at the cross-over trajectory has already been pointed out in Ref. [132]. We would like to specialize this interpretation. In the infinite momentum frame one might label the proton state by the center of plus-momentum, given as the expectation value $\boldsymbol{b}_{\perp}$ of the two generators for transversal Galilean boost [133]. The emission of a parton with momentum fraction $X=2 x /(1+x)$ leads to a change of the center of plus-momentum $\boldsymbol{b}_{\perp}^{\text {in }}$ of the initial nucleon. Since for $\eta=x$ the outgoing spectator system absorbs a quark with zero longitudinal momentum fraction, its center of plus-momentum will not change and it coincides with the one of the final state nucleon $\boldsymbol{b}_{\perp}^{\text {spec }}=\boldsymbol{b}_{\perp}^{\text {out }}$. The difference $\boldsymbol{\delta}_{\perp}=\boldsymbol{b}_{\perp}^{\text {in }}-\boldsymbol{b}_{\perp}^{\text {spec }}$ can now be viewed as the transversal distance of the struck parton from the collective spectator system. One might now adopt the GPD wave function overlap interpretation in the impact parameter space [134], which tells that the Fourier conjugate variable of $\boldsymbol{\delta} \boldsymbol{b}_{\perp}$ is simply the transverse momentum transfer $\boldsymbol{\Delta}_{\perp}$, directly observed in experiment. In other words, following the procedure of Ref. [132] gives us access to the transversal separation distance of the struck quark and collective spectator system 17 A probabilistic GPD interpretation as for the zero skewness case, however, does not hold here anymore.

Also, the skewness effect can be addressed from the experimental side. In DVCS observables for an unpolarized nucleon target one might first neglect the non-dominant CFFs $\widetilde{\mathcal{H}}, \mathcal{E}$ and $\widetilde{\mathcal{E}}$ as well as twist-three contributions. This seems to be justified to a certain extent, at least for HERMES kinematics. For the dominant quantity $\mathcal{H}$ the corresponding GPDs in the zero skewness case are well constrained by PDFs and form factors. Guidance from model calculations and lattice data might be employed with care, too, to set up field theory inspired GPD models for the zero skewness case. The skewness function, which relates the GPD for zero skewness to the one on the

\footnotetext{
${ }^{17}$ This interpretation has been also presented in Ref. 135, including considerations on the $t$-behavior of GPDs at large $\eta=x$ as it arises also from power-like wave function models [69, 70, 71.
} 
cross-over trajectory is constrained by GPD sum rules (GPDSR). It can then directly be fitted to experimental data. The limitation of this pragmatical approach for the collider experiments $\mathrm{H} 1$ and ZEUS is also obvious: the perturbative prediction of the scale change, which gives us an additional handle on the skewness effect, cannot be implemented. Since the functional form of CFFs and GPDs drastically simplify in this kinematics, experimental data can be analyzed in the traditional manner within a flexible GPD parameterization in the small $x$ region. This can be done in any GPD representation; however, it has up to now only been applied in the conformal approach.

We expect that a global LO analysis of present DVCS data can already provide a generic understanding of the GPD at the cross-over trajectory, in particular for the dominant GPD $H$. Once this understanding is reached one can address the deconvolution problem, i.e., to find a realistic parameterization of GPD models. This is particularly important if in dedicated experiments the CFFs $\mathcal{E}$ and $\widetilde{\mathcal{E}}$ are measured and one wants to access the GPDs $E$ and $\widetilde{E}$, where the former one gives access to the quark angular momentum. Realistic and flexibly parameterized GPD models should then open the road to the inclusion of radiative corrections and the Wandzura-Wilczek approximation of twist-three GPDs, see, e.g., Ref. [136, 137], or to address quark-gluon-quark GPDs [138]. If experimental data for virtual electroproduction of mesons are analyzed within the GPD formalism, it might be inevitable to go to next-to-leading order accuracy. The formalism has been worked out for the twist-two sector [107, 108, 109, 139, 140].

In this paper it has clearly been spelled out that GPDs have dual properties. If CFFs possess Regge behavior, this duality relation is perfect and the GPDs contain the same information in the central and outer region. This duality property is implemented in a GPDSR family and can be expressed in terms of GPD moments, too. Unfortunately, these GPDSRs are not sufficient to find a unique GPD model from (ideal) experimental DVCS measurements. There are two possible ways to get an handle on the remaining degrees of freedoms.

The first way relies on the common QCD tools: phenomenological knowledge, counting rules, model calculations, and lattice simulations. Certainly, lattice simulations can only provide a flash on the skewness effect, while model calculations suffer from the matching problem of partonic and collective degrees of freedom. A central theoretical task here is to understand the small $x$ behavior of GPDs. It seems to be rather promising to study the $t$-channel ladder, e.g., to proceed along the lines of Refs. [44] or [117]. We emphasize that phenomenological knowledge can be implemented from the $t$-channel point of view for small $x$ and also from the $s$-channel point of view for large $x$, where both regions are tied together by GPDSR. This suggests to look for dual GPD models in which the meson-baryon duality conjecture, employed in the phenomenology of hadronic onshell processes, is implemented. Crossing relations via the Froissart-Gribov projection provide an 
additional phenomenological handle.

The second way to address the problem is to develop a theoretical understanding of whether there is some holographic principle that tells us how the information about the GPD on the cross-over trajectory is related to its values in the central (or outer) region, which includes the $\eta=0$ case. This question arises naturally if one relates the partonic GPD interpretation to the $t$ - and $s$-channel hadronic point of view. We recall that the conjecture of duality originated in experimental findings about hadronic on-shell processes and was extensively studied within FESR; see, e.g., Ref. [52]. Thereby, the $\mathrm{SO}(3)$ partial wave expansions in both the $t$ - and $s$-channel were utilized in the context of Regge phenomenology, which provides an empirical description of the strong interaction phenomena. It was also realized that duality phenomena could be partially explained within the quark model. In dual hadronic resonance models the underlying symmetry of duality is the $\mathrm{SO}(2,1)$ group. Finally, the quark model was gauged providing us QCD, and string theory was born out of dual hadronic resonance models, i.e., out of the group $\mathrm{SO}(2,1)$. Certainly, in QCD phenomenology quark-hadron duality, in particular duality between the deep-inelastic and the resonance kinematical regime (Bloom-Gilman duality) is an important phenomenological concept; for a review see Ref. [141. Also conformal symmetry shows up in various QCD corners 142, 143, 144, 145, 146, 58, and integrability phenomena in the multi-color limit were discovered in QCD [147, 148, 149, 150, 151] and in supersymmetric Yang-Mill theories; see, e.g., Refs. [152, 153] and references therein. These integrability phenomena play a key role in getting a handle on the AdS/CFT conjecture [53, 54, 55]. Initiated by this conjecture, model dependent approaches to a supposed AdS/QCD duality were developed [154, 155, 156], see also Refs. [157, 158, and its application to high-energy physics is discussed, e.g., in Ref. [159]. From this more historical line, or, better to say, loop, we believe that it is rather worth it to study GPDs from the AdS/QCD point of view. Certainly, it is not expected that there is an exact answer for (scheme dependent) partonic quantities; however, one might perhaps find guidelines for the parameterization of GPDs with respect to their skewness dependence.

\section{Acknowledgements}

D.M. is grateful to the Rudjer Bošković Institute and the Department of Physics at the University of Zagreb for an invitation and the warm hospitality during his stay, during which this work has been completed. He likes to thank his colleagues from Bochum for numerous fruitful discussions, in particular, K. Goeke, P. Pobylitsa, M. Polyakov, and P. Schweitzer. For discussion on different aspects in this paper we would like to thank I.V. Anikin, S. Brodsky, M. Diehl, L. Favart, M. Furić, M. Guidal, V. Guzey, Ph. Hägler, P. Kroll, J. Noritzsch, A. V. Radyushkin, A. Schäfer, L. Schoeffel, O. V. Teryaev and M. Vanderhaeghen. K.K. and D.M. would also like to thank B. Pire and 
F. Sebastian for invitation to the Journées GDR Nucléon meeting "Extracting GPDs", where this work has been presented in a preliminary form. K.K. would like to thank the Institut für Theoretische Physik II at Ruhr-Universität Bochum for a kind hospitality. This work was supported in part by the BMBF (Federal Ministry for Education and Research), contract FKZ 06 B0 103 and by the Croatian Ministry of Science, Education and Sport under contracts no. 119-0982930-1016 and 098-0982930-2864.

\section{References}

[1] D. Müller, D. Robaschik, B. Geyer, F.-M. Dittes and J. Hořejši, Fortschr. Phys. 42, 101 (1994), [hep-ph/9812448].

[2] A. V. Radyushkin, Phys. Lett. B380, 417 (1996), hep-ph/9604317].

[3] X. Ji, Phys. Rev. D55, 7114 (1997), hep-ph/9609381].

[4] J. Collins, L. Frankfurt and M. Strikman, Phys. Rev. D56, 2982 (1997), hep-ph/9611433.

[5] J. Collins and A. Freund, Phys. Rev. D59, 074009 (1999), hep-ph/9801262.

[6] M. Diehl, Phys. Rept. 388, 41 (2003), hep-ph/0307382].

[7] A. V. Belitsky and A. V. Radyushkin, Phys. Rept. 418, 1 (2005), hep-ph/0504030.

[8] X. Ji, Phys. Rev. Lett. 78, 610 (1997), hep-ph/9603249].

[9] H1, C. Adloff et al., Phys. Lett. B517, 47 (2001), hep-ex/0107005.

[10] HERMES, A. Airapetian et al., Phys. Rev. Lett. 87, 182001 (2001), hep-ex/0106068.

[11] CLAS, S. Stepanyan, Phys. Rev. Lett. 87, 182002 (2001), hep-ex/0107043.

[12] ZEUS, S. Chekanov et al., Phys. Lett. B573, 46 (2003), hep-ex/0305028.

[13] H1, A. Aktas et al., Eur. Phys. J. C44, 1 (2005), hep-ex/0505061.

[14] Jefferson Lab Hall A and Hall A DVCS, C. M. Camacho et al., Phys. Rev. Lett. 97, 262002 (2006), nucl-ex/0607029].

[15] HERMES, A. Airapetian et al., Phys. Rev. D75, 011103 (2007), hep-ex/0605108.

[16] CLAS, S. Chen et al., Phys. Rev. Lett. 97, 072002 (2006), hep-ex/0605012. 
[17] Jefferson Lab Hall A, M. Mazouz et al., Phys. Rev. Lett. 99, 242501 (2007), [0709.0450 [nucl-ex]].

[18] H1, F. D. Aaron et al., Phys. Lett. B659, 796 (2008), [0709.4114 [hep-ex]].

[19] CLAS, F. X. Girod et al., Deeply Virtual Compton Scattering Beam-Spin Asymmetries, 0711.4805 [hep-ex], 2007.

[20] HERMES, A. Airapetian et al., Measurement of Azimuthal Asymmetries With Respect To Both Beam Charge and Transverse Target Polarization in Exclusive Electroproduction of Real Photons, 0802.2499 [hep-ex], 2008.

[21] ZEUS, J. Breitweg et al., Eur. Phys. J. C6, 603 (1999), hep-ex/9808020.

[22] H1, C. Adloff et al., Eur. Phys. J. C13, 371 (2000), [hep-ex/9902019].

[23] ZEUS, J. Breitweg et al., Eur. Phys. J. C12, 393 (2000), hep-ex/9908026].

[24] HERMES, A. Airapetian et al., Phys. Lett. B659, 486 (2008), [0707.0222 [hep-ex]].

[25] M. Guidal and S. Morrow, Exclusive $\rho^{0}$ electroproduction on the proton: GPDs or not GPDs?, 0711.3743 [hep-ph], 2007.

[26] M. Vanderhaeghen, P. A. M. Guichon and M. Guidal, Phys. Rev. Lett. 80, 5064 (1998).

[27] A. V. Belitsky, D. Müller and A. Kirchner, Nucl. Phys. B629, 323 (2002), hep-ph/0112108.

[28] A. Kirchner and D. Müller, Eur. Phys. J. C32, 347 (2003), hep-ph/0302007].

[29] V. Guzey and T. Teckentrup, Phys. Rev. D74, 054027 (2006), hep-ph/0607099].

[30] M. V. Polyakov and M. Vanderhaeghen, Taming Deeply Virtual Compton Scattering, 0803.1271 [hep-ph], 2008.

[31] A. Freund and M. F. McDermott, Phys. Rev. D65, 091901 (2002), hep-ph/0106124.

[32] A. Freund and M. McDermott, Eur. Phys. J. C23, 651 (2002), hep-ph/0111472.

[33] K. Kumerički, D. Müller, K. Passek-Kumerički and A. Schäfer, Phys. Lett. B 648, 186 (2007), [hep-ph/0605237].

[34] K. Kumerički, D. Müller and K. Passek-Kumerički, Nucl. Phys. B 794, 244 (2008), hep-ph/0703179. 
[35] J. M. Laget, Phys. Lett. B489, 313 (2000), hep-ph/0003213.

[36] F. Cano and J. M. Laget, Phys. Lett. B551, 317 (2003), [hep-ph/0209362], Erratum-ibid. B571 (2003) 250.

[37] M. Capua, S. Fazio, R. Fiore, L. Jenkovszky and F. Paccanoni, Phys. Lett. B645, 161 (2007), hep-ph/0605319.

[38] J. M. Laget, Phys. Rev. C76, 052201 (2007), [0708.1250 [hep-ph]].

[39] K. Kumerički, D. Müller and K. Passek-Kumerički, Fitting DVCS at NLO and beyond, 0710.5649 [hep-ph], 2007.

[40] M. V. Polyakov, Phys. Lett. B659, 542 (2008), [0707.2509 [hep-ph]].

[41] M. V. Polyakov, Educing GPDs from amplitudes of hard exclusive processes, 0711.1820 [hep-ph], 2007.

[42] D. Müller and A. Schäfer, Nucl. Phys. B739, 1 (2006), hep-ph/0509204].

[43] O. V. Teryaev, Analytic properties of hard exclusive amplitudes, hep-ph/0510031, 2005.

[44] L. Frankfurt, A. Freund, V. Guzey and M. Strikman, Phys. Lett. B418, 345 (1998), [hep-ph/9703449], Erratum-ibid. B429 (1998) 414.

[45] Z. Chen, Nucl. Phys. B525, 369 (1998), [hep-ph/9705279].

[46] M. Diehl and D. Y. Ivanov, Eur. Phys. J. C52, 919 (2007), [0707.0351 [hep-ph]].

[47] I. V. Anikin and O. V. Teryaev, Phys. Rev. D76, 056007 (2007), [0704.2185 [hep-ph]].

[48] P. V. Pobylitsa, Phys. Rev. D66, 094002 (2002), hep-ph/0204337.

[49] P. V. Pobylitsa, Phys. Rev. D70, 034004 (2004), hep-ph/0211160.

[50] P. V. Pobylitsa, Phys. Rev. D67, 034009 (2003), hep-ph/0210150.

[51] A. Freund, Phys. Lett. B472, 412 (2000), hep-ph/9903488].

[52] P. D. B. Collins, An Introduction to Regge Theory and High Energy Physics (Cambridge University Press, Cambridge, 1977).

[53] J. M. Maldacena, Adv. Theor. Math. Phys. 2, 231 (1998), hep-th/9711200. 
[54] S. S. Gubser, I. R. Klebanov and A. M. Polyakov, Phys. Lett. B428, 105 (1998), hep-th/9802109.

[55] E. Witten, Adv. Theor. Math. Phys. 2, 253 (1998), hep-th/9802150.

[56] M. Guidal, Discussion on the recent proton-DVCS results of Jefferson Lab, 0803.1592 [hep-ph], 2008.

[57] A. V. Radyushkin, Phys. Rev. D56, 5524 (1997), hep-ph/9704207.

[58] V. M. Braun, G. P. Korchemsky and D. Müller, Prog. Part. Nucl. Phys. 51, 311 (2003), hep-ph/0306057.

[59] A. V. Belitsky, B. Geyer, D. Müller and A. Schäfer, Phys. Lett. B421, 312 (1998), hep-ph/9710427.

[60] A. G. Shuvaev, Phys. Rev. D60, 116005 (1999), hep-ph/9902318.

[61] J. D. Noritzsch, Phys. Rev. D62, 054015 (2000), hep-ph/0004012.

[62] I. I. Balitsky and V. M. Braun, Nucl. Phys. B311, 541 (1989).

[63] N. Kivel and L. Mankiewicz, Nucl. Phys. B557, 271 (1999), hep-ph/9903531.

[64] A. Manashov, M. Kirch and A. Schäfer, Phys. Rev. Lett. 95, 012002 (2005), hep-ph/0503109.

[65] M. Kirch, A. Manashov and A. Schäfer, Phys. Rev. D72, 114006 (2005), hep-ph/0509330.

[66] M. Diehl, T. Feldmann, R. Jakob and P. Kroll, Eur. Phys. J. C8, 409 (1999), hep-ph/9811253.

[67] S. J. Brodsky, M. Diehl and D. S. Hwang, Nucl. Phys. B596, 99 (2001), hep-ph/0009254.

[68] M. Diehl, T. Feldmann, R. Jakob and P. Kroll, Nucl. Phys. B596, 33 (2001), hep-ph/0009255, Erratum-ibid. B605 (2001) 647.

[69] A. Mukherjee, I. V. Musatov, H. C. Pauli and A. V. Radyushkin, Phys. Rev. D67, 073014 (2003), hep-ph/0205315.

[70] B. C. Tiburzi, W. Detmold and G. A. Miller, Phys. Rev. D70, 093008 (2004), hep-ph/0408365.

[71] D. S. Hwang and D. Müller, Phys. Lett. B660, 350 (2008), [0710.1567 [hep-ph]]. 
[72] A. V. Radyushkin, Phys. Rev. D59, 014030 (1999), hep-ph/9805342.

[73] A. V. Radyushkin, Phys. Lett. B449, 81 (1999), hep-ph/9810466].

[74] K. Goeke, M. V. Polyakov and M. Vanderhaeghen, Prog. Part. Nucl. Phys. 47, 401 (2001), hep-ph/0106012.

[75] A. V. Radyushkin, Phys. Rev. D58, 114008 (1998), hep-ph/9803316.

[76] S. Boffi and B. Pasquini, Generalized parton distributions and the structure of the nucleon, 0711.2625 [hep-ph], 2007.

[77] LHPC and SESAM, P. Hägler et al., Phys. Rev. D68, 034505 (2003), hep-lat/0304018.

[78] LHPC, P. Hägler et al., Eur. Phys. J. A24S1, 29 (2005), hep-ph/0410017].

[79] LHPC and SESAM, P. Hägler et al., Phys. Rev. Lett. 93, 112001 (2004), hep-lat/0312014.

[80] QCDSF, M. Göckeler et al., Phys. Rev. Lett. 92, 042002 (2004), hep-ph/0304249.

[81] QCDSF and UKQCD, M. Göckeler et al., Phys. Lett. B627, 113 (2005), hep-lat/0507001.

[82] R. G. Edwards et al., PoS LAT 2006, 121 (2006), hep-lat/0610007.

[83] LHPC, P. Hägler et al., Nucleon Generalized Parton Distributions from Full Lattice QCD, 0705.4295 [hep-lat], 2007.

[84] M. V. Polyakov and A. G. Shuvaev, On 'dual' parametrizations of generalized parton distributions, hep-ph/0207153, 2002.

[85] M. Diehl, T. Gousset, B. Pire and O. V. Teryaev, Phys. Rev. Lett. 81, 1782 (1998), hep-ph/9805380].

[86] O. V. Teryaev, Phys. Lett. B510, 125 (2001), hep-ph/0102303.

[87] F.-M. Dittes, D. Müller, D. Robaschik, B. Geyer and J. Hořejši, Phys. Lett. 209B, 325 (1988).

[88] K. Kumerički, D. Müller, K. Passek-Kumerički and A. Schäfer, In preparation.

[89] S. Ahmad, H. Honkanen, S. Liuti and S. K. Taneja, Generalized parton distributions from hadronic observables, 0709.0016 [hep-ph], 2007.

[90] F. J. Yndurain, Phys. Lett. B 74, 68 (1978). 
[91] S. Ahmad, H. Honkanen, S. Liuti and S. K. Taneja, Phys. Rev. D75, 094003 (2006), hep-ph/0611046].

[92] A. V. Belitsky, D. Müller, L. Niedermeier and A. Schäfer, Phys. Lett. B474, 163 (2000), hep-ph/9908337.

[93] L. Mankiewicz, G. Piller and A. Radyushkin, Eur. Phys. J. C 10, 307 (1999), hep-ph/9812467].

[94] M. V. Polyakov and C. Weiss, Phys. Rev. D60, 114017 (1999), hep-ph/9902451.

[95] W. I. Weisberger, Phys. Rev. D5, 2600 (1972).

[96] S. J. Brodsky, F. E. Close and J. F. Gunion, Phys. Rev. D5, 1384 (1972).

[97] A. V. Belitsky, D. Müller, A. Kirchner and A.Schäfer, Phys. Rev. D64, 116002 (2001), hep-ph/0011314.

[98] S. V. Goloskokov and P. Kroll, Eur. Phys. J. C42, 281 (2005), hep-ph/0501242].

[99] S. V. Goloskokov and P. Kroll, Eur. Phys. J. C53, 367 (2008), [0708.3569 [hep-ph]].

[100] K. Goeke et al., Phys. Rev. D75, 094021 (2007), [hep-ph/0702030.

[101] F. Ellinghaus, DVCS at HERMES: Recent Results, 0710.5768 [hep], 2007.

[102] A. Freund, M. McDermott and M. Strikman, Phys. Rev. D67, 036001 (2003), hep-ph/0208160].

[103] W. L. van Neerven and A. Vogt, Nucl. Phys. B588, 345 (2000), hep-ph/0006154.

[104] S. Forte, Int. J. Mod. Phys. A21, 769 (2006), hep-ph/0509371.

[105] D. Y. Ivanov, Exclusive vector meson electroproduction, 0712.3193 [hep-ph], 2007.

[106] D. Müller, Pomeron dominance in deeply virtual Compton scattering and the femto holographic image of the proton, hep-ph/0605013, 2006.

[107] A. V. Belitsky and D. Müller, Phys. Lett. B417, 129 (1998), hep-ph/9709379.

[108] A. V. Belitsky and D. Müller, Nucl. Phys. B537, 397 (1999), hep-ph/9804379.

[109] A. V. Belitsky, A. Freund and D. Müller, Nucl. Phys. B574, 347 (2000), hep-ph/9912379.

[110] D. Müller, Phys. Lett. B634, 227 (2006), hep-ph/0510109. 
[111] K. Kumerički, D. Müller and K. Passek-Kumerički, Talk given at the conference Exclusive Reactions at High Momentum Transfer, New Port News, 2007, May 21-24; http://conferences.jlab.org/exclusive/talks/DMueller.pdf.

[112] J. Noritzsch, Private communication.

[113] A. G. Shuvaev, K. J. Golec-Biernat, A. D. Martin and M. G. Ryskin, Phys. Rev. D60, 014015 (1999), hhep-ph/9902410].

[114] M. Diehl and W. Kugler, Phys. Lett. B660, 202 (2008), [0711.2184 [hep-ph]].

[115] A. D. Martin, M. G. Ryskin and T. Teubner, Phys. Rev. D62, 014022 (2000), hep-ph/9912551.

[116] P. V. Landshoff and J. C. Polkinghorne, Phys. Rept. 5, 1 (1972).

[117] B. I. Ermolaev, M. Greco and S. I. Troyan, Nucl. Phys. B594, 71 (2001), hep-ph/0009037].

[118] X. Ji, J. Phys. G24, 1181 (1998), hep-ph/9807358.

[119] A. V. Afanasev and C. E. Carlson, Phys. Rev. Lett. 94, 212301 (2005), hep-ph/0502128].

[120] A. D. Polyanin and A. V. Manzhirov, Handbook of Integral Equations (CRC Press LLC, Boca Raton, USA, 1998).

[121] R. Dolen, D. Horn and C. Schmid, Phys. Rev. 166, 1768 (1968).

[122] M. J. Creutz, S. D. Drell and E. A. Paschos, Phys. Rev. 178, 2300 (1969).

[123] A. Belitsky, A. Kirchner, D. Müller and A. Schäfer, Phys.Lett. B510, 117 (2001), hep-ph/0103343].

[124] F. Carlson, Sur une classe de séries de Taylor, PhD thesis, Uppsala University, 1914.

[125] I. M. Gelfand and G. E. Shilov, Generalized Functions Vol. I (Academic Press, New York, 1964).

[126] K. M. Semenov-Tian-Shansky, Forward-like functions for dual parametrization of GPDs from nonlocal chiral quark model, 0803.2218 [hep-ph], 2008.

[127] F. E. Close and Q. Zhao, Phys. Rev. D66, 054001 (2002), hep-ph/0202181].

[128] M. Diehl, T. Feldmann, R. Jakob and P. Kroll, Eur. Phys. J. C39, 1 (2005), hep-ph/0408173. 
[129] M. Guidal, M. V. Polyakov, A. V. Radyushkin and M. Vanderhaeghen, Phys. Rev. D72, 054013 (2005), hep-ph/0410251].

[130] G. Veneziano, Nuovo. Cim. A57, 190 (1968).

[131] F. Yuan, Phys. Rev. D69, 051501 (2004), hep-ph/0311288.

[132] J. P. Ralston and B. Pire, Phys. Rev. D66, 111501 (2002), hep-ph/0110075].

[133] J. Kogut and D. Soper, Phys. Rev. D1, 2901 (1970).

[134] M. Diehl, Eur. Phys. J. C25, 223 (2002), hep-ph/0205208], Erratum-ibid. C31 (2003) 277.

[135] M. Burkardt, GPDs with $\zeta \neq 0,[0711.1881$ [hep-ph]], 2007.

[136] I. V. Anikin, B. Pire and O. V. Teryaev, Phys. Rev. D 62, 071501 (2000), hep-ph/0003203].

[137] N. Kivel and M. V. Polyakov, Nucl. Phys. B600, 334 (2001), hep-ph/0010150.

[138] A. V. Belitsky and D. Müller, Nucl. Phys. B589, 611 (2000), hep-ph/0007031.

[139] A. V. Belitsky and D. Müller, Phys. Lett. B513, 349 (2001), hep-ph/0105046].

[140] D. Y. Ivanov, L. Szymanowski and G. Krasnikov, JETP Lett. 80, 226 (2004), [hep-ph/0407207], Pisma Zh. Eksp. Teor. Fiz 80 (2004) 255.

[141] W. Melnitchouk, R. Ent and C. Keppel, Phys. Rept. 406, 127 (2005), hep-ph/0501217].

[142] S. Brodsky, Y. Frishman, G. Lepage and C. Sachradja, Phys. Lett. 91B, 239 (1980).

[143] A. Efremov and A. Radyushkin, Phys. Lett. B94, 245 (1980).

[144] L. N. Lipatov, Sov. Phys. JETP 63, 904 (1986).

[145] L. N. Lipatov, Phys. Lett. B309, 394 (1993).

[146] D. Müller, Phys. Rev. D58, 054005 (1998), [hep-ph/9704406].

[147] L. N. Lipatov, JETP Lett. 59, 596 (1994).

[148] L. D. Faddeev and G. P. Korchemsky, Phys. Lett. B342, 311 (1995), hep-th/9404173.

[149] G. P. Korchemsky, Nucl. Phys. B443, 255 (1995), hep-ph/9501232].

[150] V. M. Braun, S. E. Derkachov, G. P. Korchemsky and A. N. Manashov, Nucl. Phys. B553, 355 (1999), hep-ph/9902375]. 
[151] A. V. Belitsky, Nucl. Phys. B574, 407 (2000), hep-ph/9907420].

[152] N. Beisert, Phys. Rept. 405, 1 (2005), hep-th/0407277].

[153] A. V. Belitsky, G. P. Korchemsky and D. Mueller, Nucl. Phys. B735, 17 (2006), hep-th/0509121].

[154] S. J. Brodsky and G. F. de Teramond, Phys. Lett. B582, 211 (2004), hep-th/0310227].

[155] G. F. de Teramond and S. J. Brodsky, Phys. Rev. Lett. 94, 201601 (2005), hep-th/0501022].

[156] H. R. Grigoryan and A. V. Radyushkin, Phys. Lett. B650, 421 (2007), hep-ph/0703069.

[157] J. Polchinski and M. J. Strassler, Phys. Rev. Lett. 88, 031601 (2002), hep-th/0109174|.

[158] R. C. Brower, J. Polchinski, M. J. Strassler and C.-I. Tan, JHEP 0712, 005 (2007), hep-th/0603115].

[159] D. M. Hofman and J. Maldacena, Conformal collider physics: Energy and charge correlations, 0803.1467 [hep-th], 2008. 